\title{
CROSSED SIMPLICIAL GROUPS AND THEIR ASSOCIATED HOMOLOGY
}

\author{
ZBIGNIEW FIEDOROWICZ AND JEAN-LOUIS LODAY
}

\begin{abstract}
We introduce a notion of crossed simplicial group, which generalizes Connes' notion of the cyclic category. We show that this concept has several equivalent descriptions and give a complete classification of these structures. We also show how many of Connes' results can be generalized and simplified in this framework.
\end{abstract}

A simplicial set (resp. group) is a family of sets (resp. groups) $\left\{G_{n}\right\}_{n \geq 0}$ together with maps (resp. group homomorphisms) which satisfy some well-known universal formulas. The geometric realization of a simplicial set is a space and the geometric realization of a simplicial group is a topological group.

We define a crossed simplicial group as a simplicial set $G_{*}=\left\{G_{n}\right\}_{n \geq 0}$ such that the $G_{n}$ 's are groups and the faces and degeneracies are crossed group homorphisms, that is, satisfy a formula like

$$
f\left(g g^{\prime}\right)=f(g)(g \cdot f)\left(g^{\prime}\right)
$$

(see $\S 1$ for the precise definition). A simplicial group is thus the particular case of a trivial action. The geometric realization of a crossed simplicial group is still a topological group.

The reason for introducing such objects comes from cyclic homology whose definition, as given by Connes in [C], relies on the existence of a certain category $\Lambda$ (denoted $\Delta C$ in this paper ) satisfying some special properties. In fact these properties are equivalent to the following assertion: the standard simplicial circle can be endowed with the structure of a crossed simplicial group $C_{*}=$ $\left\{C_{n}\right\}_{n \geq 0}$ with $C_{n}=\mathbf{Z} / n+1$ (cyclic groups). In [L] we remarked that the family of dihedral groups $\left\{D_{n+1}\right\}_{n \geq 0}$ (resp. quaternion groups $\left\{Q_{n+1}\right\}_{n \geq 0}$ ) forms a crossed simplicial group (but not a simplicial group). The notion of crossed simplicial group provides a useful conceptual framework for studying these basic examples.

In this paper we investigate the existence of other families of groups bearing a crossed simplicial group structure. In particular we show that it is the case for the family of symmetric groups $S_{*}=\left\{S_{n+1}\right\}_{n \geq 0}$. Then in 3.6 we give a complete classification theorem:

Received by the editors May 28, 1988 .

1980 Mathematics Subject Classification (1985 Revision). Primary 18D05, 18F25, 18 G30. 
For any crossed simplicial group $G_{*}$ there exists a (unique up to isomorphism) exact sequence of crossed simplicial groups

$$
1 \rightarrow G_{*}^{\prime} \rightarrow G_{*} \rightarrow G_{*}^{\prime \prime} \rightarrow 1
$$

such that $G_{*}^{\prime}$ is a simplicial group and $G_{*}^{\prime \prime}$ is one of the following crossed simplicial groups: $\{1\}, C_{*}, S_{*},\{\mathbf{Z} / 2\}, D_{*}, \mathbf{Z} / 2 \times S_{*}$ and $H_{*}$. In this result $H_{n}$ denotes the hyperoctahedral group $(\mathbf{Z} / 2)^{n} \rtimes S_{n}$. The geometric realizations of these seven particular crossed simplicial groups are respectively a point, $S^{1}$, a contractible space, $\mathrm{Z} / 2, O(2), \mathbf{Z} / 2$, a contractible space.

As in cyclic homology theory, a crossed simplicial group $G_{*}$ determines a small category denoted $\Delta G$ which bears the same special properties as $\Delta C$, with $C_{n}$ replaced by $G_{n}$. We define a $G_{*}$-space as a functor $X_{*}: \Delta G^{\mathbf{o p}} \rightarrow$ Top . In particular it is a simplicial set. So there should be a relation between its geometric realization as a simplicial set $\left|X_{*}\right|$ and its geometric realization as a $G_{*}$-space hocolim ${ }_{\Delta G} X_{*}$. It is given by Theorem 5.9:

For any $G_{*}$-space $X_{*}$ there is a natural fibration sequence

$$
\left|X_{*}\right| \rightarrow \text { hocolim }_{\Delta G} X_{*} \rightarrow B \Delta G .
$$

This is the topological analogue of the periodicity exact sequence relating cyclic homology with Hochschild homology.

Cyclic homology is obtained through the functor $A^{\otimes}: \Delta C^{\mathbf{o p}} \rightarrow(k$-Mod), $[n] \rightarrow A^{\otimes n+1}$, where $A$ is a $k$-algebra, as $H C_{n}(A)=\operatorname{Tor}_{n}^{\Delta C}\left(k, A^{\otimes}\right)$. More generally any functor $M: \Delta G^{\mathbf{0 p}} \rightarrow(k$-Mod) gives rise to homology groups $H G_{n}(M)=\operatorname{Tor}_{n}^{\Delta G}(k, M)$, and we show that most of the properties of cyclic homology (like existence of spectral sequences) carry over to these new homology theories. Finally we prove that the homology of a $\Delta S^{\mathbf{0 p}}$-module considered as a simplicial module does not change in characteristic zero after taking the coinvariants by the symmetric group at each level (cf. 6.17).

The contents of this paper are as follows.

$\S 1$ contains the definition of a crossed simplicial group $G_{*}$ and some examples.

In $\S 2$ we show that the true structure underneath is a double category. This explains why spectral sequences arise naturally in this framework.

$\S 3$ contains the description of the crossed simplicial group structure of $S_{*}$ and of $H_{*}$ together with the classification theorem.

In $\S 4$ we introduce $G_{*}$-spaces and free $G_{*}$-spaces.

In $\S 5$ we prove that the geometric realization $\left|G_{*}\right|$ of $G_{*}$ is a topological group and that there is a homotopy equivalence hocolim hG $_{*} \simeq E\left|G_{*}\right| \times_{\left|G_{*}\right|}\left|X_{*}\right|$ and a fibration $\left|X_{*}\right| \rightarrow$ hocolim $_{\Delta G} X_{*} \rightarrow B \Delta G$ for any $G_{*}$-space $X_{*}$.

$\S 6$ deals with the homology of $\Delta G^{\mathrm{op}}$-modules generalizing what is known for cyclic homology. We prove the existence of spectral sequences abutting to these new homology groups. 
The second author is indebted to M. Zisman for several helpful comments.

\section{Definitions AND FIRST EXAMPLES}

Let $[n]=\{0, \ldots, n\}$ be a set with $n+1$ elements. The category $\Delta$ is the category of nondecreasing maps $[n] \rightarrow[m]$ where $[n]$ is equipped with its natural ordering. The morphisms of $\Delta$ are generated by the face maps $\delta_{i}:[n-1] \rightarrow[n]$ (misses $i$ ) and the degeneracy maps $\sigma_{i}:[n+1] \rightarrow[n]$ (hits $i$ twice), $i=0, \ldots, n$. The only automorphism of $[n]$ in $\Delta$ is the identity $\mathrm{id}_{n}$. Recall that a simplicial set $X$ is a functor $X: \Delta^{\text {op }} \rightarrow$ Sets. The image of $\phi \in \operatorname{Hom}_{\Delta}$ is usually denoted $\phi^{*}$, but we put $\delta_{i}^{*}=d_{i}$ and $\sigma_{i}^{*}=s_{i}$.

1.1. Definition. A sequence of groups $\left\{G_{n}\right\}, n \geq 0$, is a crossed simplicial group if it is equipped with the following structure. There is a small category $\Delta G$, which is part of the structure, such that

(a) the objects of $\Delta G$ are $[n], n \geq 0$,

(b) $\Delta G$ contains $\Delta$ as a subcategory,

(c) $\operatorname{Aut}_{\Delta G}([n])=G_{n}^{\text {op }}$ (opposite group of $G_{n}$ ),

(d) any morphism in $\Delta G$ can be uniquely written as a composite $\phi \cdot g$ where $\phi \in \operatorname{Hom}_{\Delta}([m],[n])$ and $g \in G_{m}^{\mathbf{o p}}$ (whence the notation $\Delta G$ ).

A morphism of crossed simplicial groups $G_{*} \rightarrow G_{*}^{\prime}$ is a morphism of categories $\Delta G \rightarrow \Delta G^{\prime}$ which is the identity when restricted to $\Delta$.

Multiplication in $G_{n}$ is denoted by $g g^{\prime}$ and composition in $\Delta G$ is denoted by $g^{\prime} \cdot g$ (hence $g g^{\prime}=g^{\prime} \cdot g$ ).

Axiom (d) implies that for any $g \in G_{n}$ and any $\phi \in \operatorname{Hom}_{\Delta}([m],[n])$ there exist a unique $\phi^{*}(g) \in G_{m}$ and a unique $g^{*}(\phi) \in \operatorname{Hom}_{\Delta}([m],[n])$ such that

$$
g \cdot \phi=g^{*}(\phi) \cdot \phi^{*}(g) \text {. }
$$

Lemma 1.3. $G_{*}$ is a simplicial set.

Proof. The functor $[n] \mapsto G_{n}$ which sends $\phi \in \operatorname{Hom}_{\Delta}([m],[n])$ to $\phi^{*}: G_{n} \rightarrow$ $G_{m}$ is well defined because of associativity in $\Delta G$; therefore $(\phi \cdot \psi)^{*}(g)$ $=\psi^{*}\left(\phi^{*}(g)\right)$.

Proposition 1.4. A simplicial group is a particular case of crossed simplicial group.

Proof. In order to construct the category $\Delta G$ associated to the simplicial group $G_{*}$, we need only to tell what the composite $g \cdot \phi$ is for $g \in G_{n}$ and $\phi \in \mathrm{Hom}_{\Delta}$, and to show that associativity holds. We put $g \cdot \phi=\phi \cdot \phi^{*}(g)$ where $\phi^{*}(g)$ is given by the simplicial structure of $G_{*}$. Associativity in $\Delta G$ follows from the simplicial structure. Axiom (c) is fulfilled because the only automorphisms in $\Delta$ are identities.

So this is a first example of crossed simplicial group.

1.5. Example 2. Let $G_{n}=\mathbf{Z} / 2$ with generator $y_{n+1}$ and put $y_{n+1} \cdot \delta_{i}=\delta_{n-i} \cdot y_{n}$ and $y_{n+1} \cdot \sigma_{i}=\sigma_{n-i} \cdot y_{n+2}$. One easily checks that this gives a crossed simplicial group, whose geometric realization is $\mathbf{Z} / 2$. 
Example 3. Let $G_{n}=\mathbf{Z}$ with generator $t_{n+1}$. The composition in $\Delta G$ is defined by the following formulas:

$$
\begin{gathered}
t_{n+1} \cdot \delta_{i}=\delta_{i-1} \cdot t_{n} \text { for } 1 \leq i \leq n, \quad \text { and } t_{n+1} \cdot \delta_{0}=\delta_{n}, \\
t_{n+1} \cdot \sigma_{i}=\sigma_{i-1} \cdot t_{n+2} \text { for } 1 \leq i \leq n, \quad \text { and } t_{n+1} \cdot \sigma_{0}=\sigma_{n} \cdot t_{n+2}^{2} .
\end{gathered}
$$

The geometric realization of this crossed simplicial group is homeomorphic to $\mathbf{R}$. The category $\Delta \mathbf{Z}$ associated to this crossed simplicial group $\mathbf{Z}_{*}$ is closely related to the duplicial category of [D-K].

Example 4. In [C] A. Connes has described a category $\Lambda$ which satisfies the conditions of 1.1. In this case $G_{n}=\mathbf{Z} /(n+1)$, the cyclic group of order $n+1$. This category $\Lambda$ will be denoted $\Delta C$ in this paper. It is described by the same relations as in the preceding example but with $\left(t_{n+1}\right)^{n+1}=\mathrm{id}_{n}$. Its geometric realization is homeomorphic to $S^{1}$ because the only nondegenerate simplices are $t_{1}$ and $t_{2}$. The category $\Delta C$ is a subcategory of the category $\Delta S$ which will be constructed in $\S 3$.

Example 5. Let $D_{n}=\left\{x, y \mid x^{n}=y^{2}=1\right.$ and $\left.y x y^{-1}=x^{-1}\right\}$ be the dihedral group and let $Q_{n}=\left\{x, y \mid x^{n}=y^{2}, y x y^{-1}=x^{-1}\right\}$ be the quaternion (or binary dihedral) group. Crossed simplicial groups $\left\{G_{n}\right\}$ with $G_{n}=D_{n+1}$ or $Q_{n+1}$ were described in [L] (see also $\S 3$ ). The geometric realization of $D_{*}$ (resp. $Q_{*}$ ) is the orthogonal group $O(2)$ (resp. the normalizer of $S^{1}$ in $S^{3}$ ).

Example 6. There exist crossed simplicial groups $\left\{G_{n}\right\}$ with $G_{n}=S_{n+1}$, the symmetric group, $G_{n}=H_{n+1}$, the hyperoctahedral group, and also $G_{n}=B_{n+1}$, the braid group. These examples will be studied in detail in $\S 3$; their classifying spaces are contractible by the following argument. Let $h$ be the inclusion of $S_{n}$ (resp. $H_{n}, B_{n}$ ) in $S_{n+1}$ (resp. $H_{n+1}, B_{n+1}$ ) obtained by letting it act on the $n$ first points. This inclusion satisfies $d_{i} h=h d_{i}$ for $0 \leq i \leq n$ and $d_{n+1} h=\mathrm{id}$, so this is a homotopy from the identity to the trivial map. The geometric realization of $S_{*}$ (resp. $H_{*}, B_{*}$ ) is a simply connected space with trivial homology; therefore it is contractible.

Example 7. Let $G_{*}$ be one of $C_{*}, D_{*}$ or $Q_{*}$. Define a new crossed simplicial group $G_{*}^{(k)}$ by $G_{n}^{(k)}=G_{k(n+1)-1}$. It is easily checked that this is still a crossed simplicial group whose geometric realization is a subdivision (and so homeomorphic to that) of $G_{*}$.

Proposition 1.6. In the crossed simplicial group $G_{*}$ the functions

$$
g^{*}: \operatorname{Hom}_{\Delta}([m],[n]) \rightarrow \operatorname{Hom}_{\Delta}([m],[n])
$$

for $g \in G_{n}$ and $\phi^{*}: G_{n} \rightarrow G_{m}$ for $\phi \in \operatorname{Hom}_{\Delta}([m],[n])$ satisfy the following relations:

1.h. $\left(\phi \cdot \phi^{\prime}\right)^{*}(g)=\phi^{\prime *}\left(\phi^{*}(g)\right)$,

1.v. $\left(g g^{\prime}\right)^{*}(\phi)=g^{\prime *}\left(g^{*}(\phi)\right)$, 
2.h. $g^{*}\left(\phi \cdot \phi^{\prime}\right)=g^{*}(\phi) \cdot\left(\phi^{*}(g)\right)^{*}\left(\phi^{\prime}\right)$,

2.v. $\phi^{*}\left(g g^{\prime}\right)=\phi^{*}(g)\left(g^{*}(\phi)\right)^{*}\left(g^{\prime}\right)$,

3.h. $\left(\mathrm{id}_{n}\right)^{*}(g)=g$ and $\phi^{*}\left(1_{n}\right)=1_{n}$,

3.v. $\left(1_{n}\right)^{*} \phi=\phi$ and $g^{*}\left(\mathrm{id}_{n}\right)=\mathrm{id}_{n}$.

Conversely, a family of groups $\left\{G_{n}\right\}_{n \geq 0}$ with two sets of functions $g^{*}$ and $\phi^{*}$ satisfying the relations above defines a crossed simplicial group.

Proof. Relations 1 and 2 are deduced from formula (1.2) and from associativity in the category $\Delta G$. Relations 3 are deduced from formula (1.2), the property of the identities and the fact that $\operatorname{id}_{n}=1_{n}$ in $\Delta G$.

Conversely, define the morphisms of $\Delta G$ to be the pairs $g \cdot \phi$ and let the composition be given by formula (1.2), composition in $\Delta$ and composition in $G_{n}$, i.e., $(g \cdot \phi) \cdot(h \cdot \psi)=\left(g \cdot \phi^{*}(h)\right) \cdot\left(h^{*}(\phi) \cdot \psi\right)$. Formulas 1,2 and 3 prove that $\Delta G$ is a well-defined category. The axioms of 1.1 are immediate to verify.

Proposition 1.7. A crossed simplicial group is a simplicial set $G_{*}$ such that $G_{n}$ is a group, together with a group homomorphism $G_{n} \rightarrow S_{n+1}=\operatorname{Aut}[n]$ for each $n$, such that

1. $d_{i}\left(g g^{\prime}\right)=d_{i}(g) d_{g^{-1}(i)}\left(g^{\prime}\right), s_{i}\left(g g^{\prime}\right)=s_{i}(g) s_{g^{-1}(i)}\left(g^{\prime}\right)$, and

2. the following set diagrams are commutative:

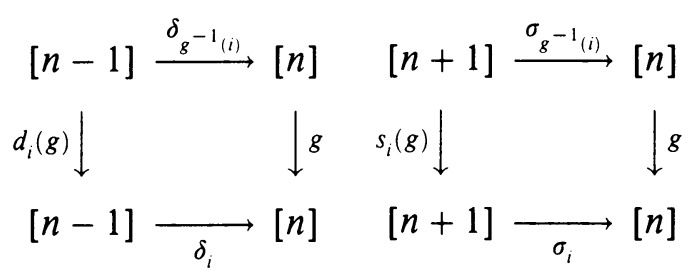

Proof. Starting with a category $\Delta G$ we already know that the family $\left\{G_{n}\right\}$ is a simplicial set and that $G_{n}$ is a group.

The element $g^{*}\left(\delta_{i}\right)$ has to be a face map $\delta_{j}$ and the set map $\rho(g):[n] \rightarrow[n]$ which sends $i$ to $j$ is a bijection. From relation $1 . v$ of 1.6 we deduce that $\rho: G_{n} \rightarrow S_{n+1}$ is an antihomomorphism, so composition with $g \mapsto g^{-1}$ gives a homomorphism. If we were dealing with degeneracies instead of faces we would have found the same representation (see [A] for detailed proofs of these facts).

Formulas 1 follow from 1.v of 1.6 and from $g^{*}\left(\delta_{i}\right)=\delta_{\rho(g)(j)}=\delta_{g^{-1}(j)}$

Formulas 2 are translations of particular cases of the identity (1.2).

On the other hand in order to construct $\Delta G$ we use Proposition 1.6. The function $\phi^{*}$ is given by the simplicial structure of $G_{*}$. The function $g^{*}$ is constructed from $g^{*}\left(\delta_{i}\right)=\delta_{g^{-1}(i)}$ and $g^{*}\left(\sigma_{i}\right)=\sigma_{g^{-1}(i)}$ by using the rule 2 .h of 1.6. To prove that this is well defined we need to verify the compatibility with the relations between faces and degeneracies. We will only do the verification for the relation $\delta_{j} \cdot \delta_{i}=\delta_{i} \cdot \delta_{j-1}$ when $i<j$.

On one hand we have $g^{*}\left(\delta_{j} \cdot \delta_{i}\right)=\delta_{g^{-1}(j)} \cdot \delta_{d_{(}(g)^{-1}(i)}$ and on the other hand $g^{*}\left(\delta_{i} \cdot \delta_{j-1}\right)=\delta_{g^{-1}(i)} \cdot \delta_{d_{i}(g)^{-1}(j-1)}$. Put $a=g^{-1}(i)$ and $b=g^{-1}(j)$. If $b<a$, 
then by property $2 d_{i}(g)^{-1}(j-1)=b$ and $d_{j}(g)^{-1}(i)=a-1$. So we need to have $\delta_{a} \cdot \delta_{b}=\delta_{b} \cdot \delta_{a-1}$, which happens to be true because $b<a$.

If $b>a$, then by property $2 d_{i}(g)^{-1}(j-1)=b-1$ and $d_{j}(g)^{-1}(i)=a$. So we need to have $\delta_{a} \cdot \delta_{b}=\delta_{b} \cdot \delta_{a-1}$, which happens to be true because $b>a$.

The other relations are proved analogously.

Relations 1, 2 and 3 of 1.6 follow from the definition of $g^{*}$ and $\phi^{*}$.

Remark 1.8. If the representations are trivial, then condition 2 is automatically fulfilled and condition 1 shows that $G_{*}$ is a simplicial group.

\section{INTERPRETATIONS IN TERMS OF DOUBLE CATEGORIES}

2.1. A small double category (cf. [ML]) is a set of objects, a set of horizontal morphisms Mor $^{\mathrm{h}}$, a set of vertical morphisms Mor ${ }^{\mathrm{v}}$ and a set of bimorphisms Bimor, together with the following structure. To any bimorphism $\alpha$ there are associated two horizontal morphisms: its horizontal source $f$ and its horizontal target $g$, and also two vertical morphisms: its vertical source $f^{\prime}$ and its vertical target $g^{\prime}$. To any horizontal (resp. vertical) morphism is associated two objects (like in an ordinary category): the source and the target. The sources and the targets of the morphisms associated to a bimorphism $\alpha$ are such that they fit into a diagram

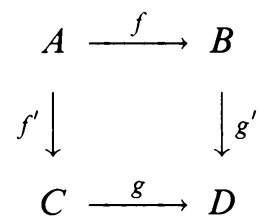

(which means $A=$ source of horizontal source $=$ source of vertical source and so on).

Moreover a double category is equipped with two composition laws: a horizontal one

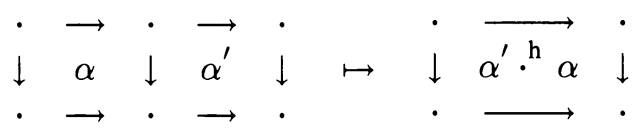

and a vertical one

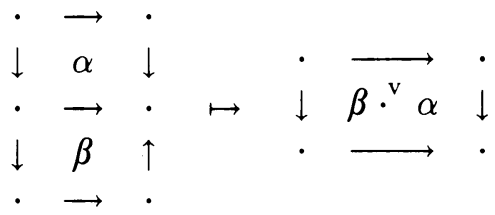

such that the horizontal (resp. vertical) composition makes (Bimor, Mor ${ }^{\mathrm{v}}$ ) (resp. (Bimor, $\left.\left.\operatorname{Mor}^{\mathrm{h}}\right)\right)$ into a category. The last requirement is the interchange law between vertical and horizontal compositions: given four composable bi- 
morphisms like below

$$
\begin{array}{lllll}
\dot{\downarrow} & \vec{\alpha} & \downarrow & \overrightarrow{\alpha^{\prime}} & \downarrow \\
\cdot & \vec{\beta} & \cdot & \overrightarrow{\beta^{\prime}} & \downarrow
\end{array}
$$

then one has the formula

$$
\left(\alpha^{\prime} \cdot{ }^{\mathrm{h}} \alpha\right) \cdot{ }^{\mathrm{v}}\left(\beta^{\prime} \cdot{ }^{\mathrm{h}} \beta\right)=\left(\beta^{\prime} \cdot{ }^{\mathrm{v}} \alpha^{\prime}\right) \cdot{ }^{\mathrm{h}}\left(\beta \cdot{ }^{\mathrm{v}} \alpha\right) .
$$

Exercise. Let $G$ be a group. Put Bimor $=G, \operatorname{Mor}^{\mathrm{h}}=\operatorname{Mor}^{\mathrm{v}}=\mathrm{Ob}=*$ (i.e., one element). Show that if horizontal and vertical compositions are given by the group law, then this is a double category if and only if $G$ is abelian.

2.2. Any small category gives rise to a simplicial set called its nerve. Similarly, a small double category gives rise to a bisimplicial set $\left(X_{p q}\right), p \geq 0$ and $q \geq$ 0 , still called its nerve. It is obtained as follows. The set $X_{p q}$ is the set of all rectangles of $p \times q$ bimorphisms which are composable according to the following picture:

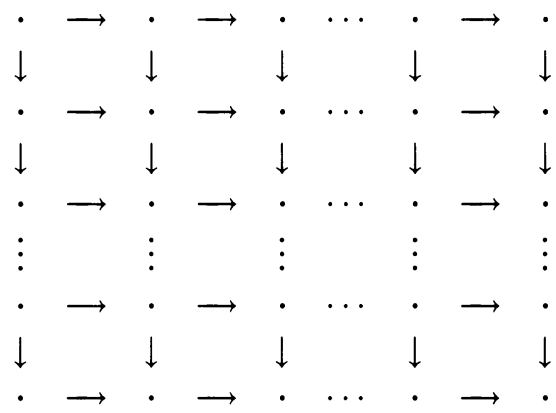

The horizontal (resp. vertical) simplicial structure is obtained as in the nerve of a category. The compatibility of the two simplicial structures follows from the interchange law in the double category. The geometric realization of this bisimplicial set is the classifying space of the double category (cf. for instance [B-K] for more details).

Exercise. In the example above the classifying space is an Eilenberg-Mac Lane space of type $K(G, 2)$.

2.3. We will now consider a special type of double category, those which satisfy the star condition:

(*) Any pair $(\phi, g)$ where $\phi$ is a horizontal morphism and $g$ a vertical morphism with the same target object determines a unique bimorphism $\alpha$.

Hence

$$
\begin{array}{cccccc} 
& & & A & \stackrel{g^{*}(\phi)}{\longrightarrow} & B \\
& & & & & \\
& & & \phi^{*}(g) \downarrow & \alpha & \downarrow g \\
\phi & D & \text { determines } & C & \underset{\phi}{\longrightarrow} & D
\end{array}
$$


As a result this pair determines a new horizontal morphism $g^{*}(\phi)$ and a new vertical morphism $\phi^{*}(g)$.

Proposition 2.4. Let $\mathbf{B}$ be a small double category which satisfies (*). Then the functions horizontal source $(\phi, g) \mapsto g^{*}(\phi)$ and vertical source $(\phi, g) \mapsto \phi^{*}(g)$ satisfy formulas 1,2 and 3 of 1.6 .

Proof. Horizontal composition and the star condition imply formula 1 .h and 2.h. Composition with the identity of the horizontal law implies 3.h. Similarly formulas 1.v, 2.v and 3.v are implied by the properties of the vertical composition.

All the examples given in the previous section give rise to a double category of the following kind. The objects are $[n], n \geq 0$, the horizontal morphisms are those of $\Delta$, the vertical morphisms are such that $\operatorname{Hom}^{\mathrm{v}}([m],[n])=\varnothing$ if $n \neq m$ and $\operatorname{Hom}^{\mathrm{v}}([n],[n])=G_{n}$ (as a group), and the bimorphisms satisfy condition $(*)$.

2.5. Let $\mathbf{B}$ be a small double category satisfying $(*)$. To $\mathbf{B}$ we associate canonically a category $\underline{B}$ as follows. The objects of $\underline{B}$ are those of $\mathbf{B}$. A morphism of $\underline{B}$ from $A$ to $C$ is a pair of morphisms

$$
A \stackrel{h}{\longleftarrow} C \stackrel{\phi}{\longrightarrow} B
$$

where $h$ is a vertical morphism and $\phi$ a horizontal morphism in B. Composition in $B$ is defined by the following diagram:

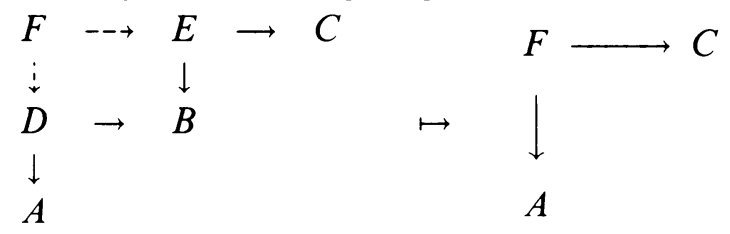

The star condition ensures that the composition is associative and has an identity.

Proposition 2.6. Let $\mathbf{B}$ be a small double category satisfying (*). Then the classifying space of $\mathbf{B}$ is canonically homotopy equivalent to the classifying space of $\underline{B}$.

Proof. The classifying space of $\mathbf{B}$ is the geometric realization of nerve $\mathbf{B}$, which is a bisimplicial set. By a classical lemma (cf. [Q, Chapter 1]) this space is homotopy equivalent to the geometric relaization of the diagonal of nerve $\mathbf{B}$. This last simplicial set is in fact, thanks to the star condition, the nerve of $\underline{B}$. As a result the classifying spaces of $\mathbf{B}$ and $\underline{B}$ are homotopy equivalent.

2.7. Remark. The construction of $\underline{B}$ looks like the $Q$-construction of Quillen [Q]. In fact any exact category $E$ determines a double category $\mathbf{Q E}$ whose bimorphisms are commutative bicartesian squares with monomorphisms (resp. epimorphisms) as horizontal (resp. vertical) morphisms. Quillen's $Q$ construction is the category $\underline{Q E}$ associated to the double category $\mathbf{Q E}$ (see $\mathbf{p}$. 201 of loc.cit). 
Proposition 2.8. A crossed simplicial group $G_{*}$ is equivalent to a double category which satisfies the $(*)$ condition, whose category of horizontal morphisms is $\Delta$ and whose category of vertical morphisms is the groupoid $\bigcup_{n} G_{n}$.

Proof. We start with the characterization of a crossed simplicial group given in Proposition 1.6. A bimorphism in $\Delta \mathbf{G}$ is of the form given in 2.3. Horizontal (resp. vertical) composition is forced by the composition in $\Delta$ (resp. in $G_{n}$ ), it is associative thanks to formula 1.h and 2.h (resp. 1.v and 2.v) and has an identity thanks to formula 3.h (resp. 3.v).

Proposition 2.9. The classifying space of the double category $\mathbf{\Delta G}$ associated to the crossed simplicial group $G_{*}$ is homeomorphic to the classifying space $B\left|G_{*}\right|$ of the topological group $\left|G_{*}\right|$.

Proof. The nerve of $\Delta \mathbf{G}$ is the bisimplicial set of rectangles (cf. 2.2). Its classifying space can be obtained by taking first the horizontal geometric realization (which gives a simplicial space) and then taking the vertical geometric realization. In the case at hand, for fixed $q$, the horizontal simplicial set is the nerve of the category associated to the functor $\Delta^{\mathrm{op}} \rightarrow$ Sets, $[n] \mapsto\left(G_{n}\right)^{q}$ (its objects are $([n], x)$ with $\left.x \in\left(G_{n}\right)^{q}\right)$. The classifying space of this category is homeomorphic to $\left|G_{*}\right|^{q}$, which is a topological group (cf. Theorem 5.3). Under these homeomorphisms the simplicial structure of $[q] \mapsto\left|G_{*}\right|^{q}$ is identified with the simplicial structure of nerve $\left|G_{*}\right|$, whence the assertion.

Remarks. The simplicial structure of $[n] \mapsto\left(G_{n}\right)^{q}$ is not the product of the simplicial structure for $q=1$, unless $G_{*}$ is a simplicial group. Bisimplicial sets lead naturally to spectral sequences; this is why it is interesting to know that the classifying space of $B$ is homotopy equivalent to the classifying space of a bisimplicial set. This will be used in Theorems 6.9 and 6.16 below.

\section{EXAMPLES AND CLASSIFICATION OF CROSSED SIMPLICIAL GROUPS}

3.1. The symmetric group $S_{n+1}$ acts on $[n]=\{0,1, \ldots, n\}$ by permutations. It will be helpful to think of a permutations $g$ as a graph linking points of $[n]$ to the points of another copy of $[n]$ :

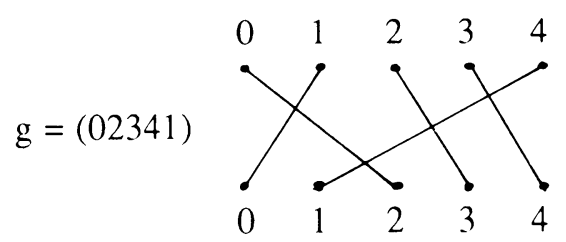

The hyperoctahedral group $H_{n+1}=(\mathbf{Z} / 2)^{n+1} \rtimes S_{n+1}$ is a semidirect product where $S_{n+1}$ acts by permutation of variables on $(\mathbf{Z} / 2)^{n+1}$. It is often denoted $S_{n+1} \int \mathbf{Z} / 2$ (wreath product). An element $g$ of $H_{n+1}$ can be viewed as a graph with labels. The edge abutting at $i$ is labelled by 1 or $y$ (generator of $\mathbf{Z} / 2$ ) according to whether the $i$ th component of $g$ in $(\mathbf{Z} / 2)^{n+1}$ is the identity or the generator. We will refer to elements of $H_{n+1}$ as labelled permutations. 
Accordingly it is helpful to think of elements of $\mathrm{Hom}_{\Delta}$, which are set maps which preserve the order, as graphs linking elements of $[\mathrm{m}]$ to their image in $[n]$.

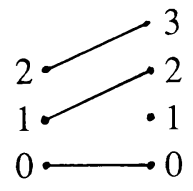

$\delta_{1}$

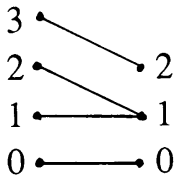

$\sigma_{1}$

Our aim is to describe a crossed simplicial group structure on $H_{n+1}$.

Description of $g^{*}(\phi)$. A map $\phi \in \mathrm{Hom}_{\Delta}$ is completely determined by the sequence of numbers $\# \phi^{-1}(i)$ for $i=0, \ldots, n$ (\# means number of elements of). Consider $g$ as a permutation of the set $[n]$ and define a new sequence of numbers by $i \mapsto \# \phi^{-1}(g(i))$. This, in turn, determines a morphism in $\Delta$ that we denote by $g^{*}(\phi) \in \operatorname{Hom}_{\Delta}([m],[n])$.

Description of $\phi^{*}(g)$. We consider $g \in H_{n+1}$ as a labelled graph. $\phi^{*}(g)$ is a labelled graph constructed from $g$ as follows. If $i$ is not hit by $g$ we delete the edge abutting to $i$. If $\# \phi^{-1}(i)=k$ (with $k \geq 1$ ) we replace the edge from $g^{-1}(i)$ to $i$ in $g$ by $k$ edges. If the label of the former edge was 1 , then these $k$ new edges are all parallel and adjacent, with labels 1 . If the label of the former edge was $y$, these $k$ new edges are as in the following picture, with labels $y$.

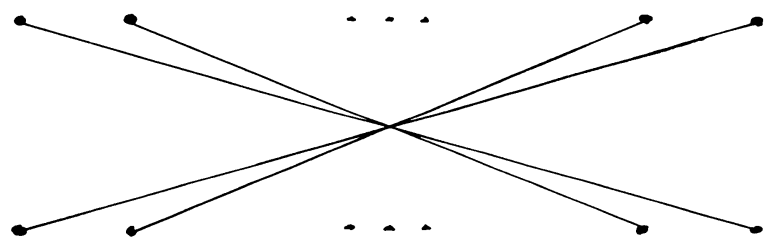

Example.

$\mathrm{g}^{*}(\phi)$

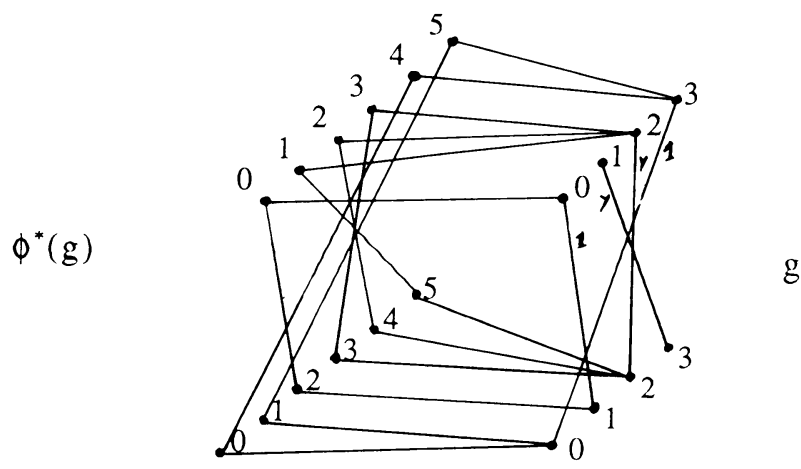


In other words the permutation $\phi^{*}(g)$ sends the subset $g^{*}(\phi)^{-1}(j)$ to the subset $\phi^{-1}(g(j))$. If the label of the edge from $g$ to $g(j)$ has label 1 then $\phi^{*}(g)$ respects the order (and the labels are 1). If the label is $y$ then $\phi^{*}(g)$ inverts the order (and the labels are $y$ ).

Lemma 3.2. Let $\phi$ be in $\operatorname{Hom}_{\Delta}([m],[n])$ and $g$ in $H_{n+1}$. Then $\phi^{*}(g)$ and $g^{*}(\phi)$ are the only elements such that the set diagram

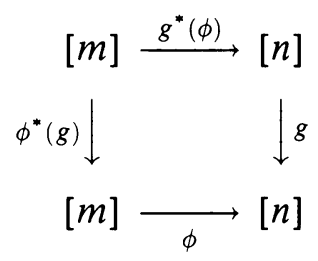

is commutative and that $\phi^{*}(g)$ preserves (resp. inverts) the order of the subsets $g^{*}(\phi)^{-1}(j)$ for $j=0,1, \ldots, n$ if the corresponding label is 1 (resp. $y$ ).

Proof. The description of $g^{*}(\phi)$ follows immediately from the following three conditions:

(a) the set diagram is commutative,

(b) $g^{*}(\phi)$ is a nondecreasing map,

(c) $\phi^{*}(g)$ is a bijection.

The description of $\phi^{*}(g)$ follows from conditions (a)-(c), and the order condition on $g^{*}(\phi)^{-1}(j)$.

Theorem 3.3. The family of hyperoctahedral groups $\left\{H_{n+1}\right\}, n \geq 0$, is a crossed simplicial group.

Proof. We remark that a picture like the example above is geometric description of a bimorphism in the double category $\Delta \mathbf{H}$.

Formulas $1 . \mathrm{h}$ and 2 .h of 1.6 come from horizontal composition in $\Delta \mathbf{H}$. Formally these formulas are proved as follows. By Lemma $3.2\left(\phi \cdot \phi^{\prime}\right)^{*}(g)$ and $g^{*}\left(\phi \cdot \phi^{\prime}\right)$ are the only elements which make the set diagram (with $g$ and $\left.\phi \cdot \phi^{\prime}\right)$ commute and which verify the order condition. So it suffices to check that $\phi^{\prime *}\left(\phi^{*}(g)\right)$ and $g^{*}(\phi) \cdot\left(\phi^{*}(g)\right)^{*}\left(\phi^{\prime}\right)$ also do. The commutativity of the square is immediate as a composite of two commutative squares. If the order of $g^{*}(\phi)^{-1}(i)$ is preserved (resp. inverted) then the labels of the corresponding edges are 1 (resp. $y$ ). Therefore the order of $\phi^{*}(g)^{*}\left(\phi^{\prime}\right)^{-1}(j)$ (for $\left.j \in g^{*}(\phi)^{-1}(i)\right)$ is also preserved (resp. inverted). The nontrivial part in this last statement comes from the following point. If $A_{1}, \ldots, A_{k}$ are ordered subsets of consecutive elements of $[n]\left(\bigcup_{i} A_{i}=[n]\right)$ then the global involution on $[n]$ is obtained by performing the involution among the subsets $A_{i}$ (which gives $\left.A_{k}, \ldots, A_{1}\right)$ and then performing the involution inside the $A_{i}$ 's.

The labels of $\phi^{\prime *}\left(\phi^{*}(g)\right)$ are determined by the labels of $g$ via $\phi \cdot \phi^{\prime}$; therefore they are the same as the labels of $\left(\phi \cdot \phi^{\prime}\right)^{*}(g)$.

Formulas $1 . \mathrm{v}$ and 2.v come from the vertical composition in $\Delta \mathbf{H}$ and they are proved similarly. 
Formulas 3.h and 3.v are immediate to verify.

Proposition 3.4. The family of groups $C_{*}=\{\mathbf{Z} / n+1\}, S_{*}=\left\{S_{n+1}\right\},\{\mathbf{Z} / 2\}$, $D_{*}=\left\{D_{n+1}\right\}$ and $\mathbf{Z} / 2 \times S_{*}$ are crossed simplicial groups.

Proof. All these groups are subgroups of $H_{n+1}$ as follows.

Let $\zeta_{n+1}$ be the product of all the $\mathbf{Z} / 2$-generators in $(Z / 2)^{n+1}$, let $\tau_{n+1}$ be the cycle $\left(\begin{array}{lll}0 & 1 & \ldots n\end{array}\right)$ in $S_{n+1}$ and let $\omega_{n+1}$ be the involution

$$
(0 n)(1 n-1)(2 n-2) \cdots
$$

in $S_{n+1}$.

In $H_{n+1}$ we verify easily that

- $\left(1, \tau_{n+1}\right)$ generates $\mathbf{Z} / n+1$,

$-S_{n+1}$ is naturally a subgroup,

$-\left(\zeta_{n+1}, \omega_{n+1}\right)$ generates $\mathbf{Z} / 2$,

- $\left(1, \tau_{n+1}\right)$ and $\left(\zeta_{n+1}, \omega_{n+1}\right)$ generate $D_{n+1}$.

We check that these families of subgroups of $H_{*}$ are stable under the action of $\phi, \phi \in \mathrm{Hom}_{\Delta}$.

For $S_{*}$ it is immediate because if all the labels of $g$ are 1 , then so are all the labels of $\phi^{*}(g)$.

For $C_{*}$ we compute $\phi^{*}(g)$ for $\phi=\delta_{i}$ and $\phi=\sigma_{i}$ and for $g=\tau_{n+1}$. Formula 1.2 for these cases gives

$$
\begin{gathered}
\tau_{n+1} \cdot \delta_{i}=\delta_{i-1} \cdot \tau_{n}, \quad 1 \leq i \leq n, \quad \tau_{n+1} \cdot \delta_{0}=\delta_{n}, \\
\tau_{n+1} \cdot \sigma_{i}=\sigma_{i-1} \cdot \tau_{n+2}, \quad 1 \leq i \leq n, \quad \tau_{n+1} \cdot \sigma_{0}=\sigma_{n} \cdot \tau_{n+2}^{2}, \mathbf{Z} / 2 \times S_{*},
\end{gathered}
$$

which shows that $\phi^{*}\left(\tau_{n+1}\right)$ is always in $C_{*}$.

For $\{\mathbf{Z} / 2\}$ we compute

$$
\begin{array}{cr}
\left(\zeta_{n+1}, \omega_{n+1}\right) \cdot \delta_{i}=\delta_{n-i} \cdot\left(\zeta_{n}, \omega_{n}\right), & 0 \leq i \leq n \\
\left(\zeta_{n+1}, \omega_{n+1}\right) \cdot \sigma_{i}=\sigma_{n-i} \cdot\left(\zeta_{n+2}, \omega_{n+2}\right), & 0 \leq i \leq n
\end{array}
$$

which shows that $\phi^{*}\left(\zeta_{n+1}, \omega_{n+1}\right)$ is always in $\{\mathbf{Z} / 2\}$.

The case $D_{*}$ is a consequence of the cases $C_{*}$ and $\{\mathbf{Z} / 2\}$.

The case $\mathbf{Z} / 2 \times S_{*}$ is a consequence of the cases $\{\mathbf{Z} / 2\}$ and $S_{*}$.

Proposition 3.5 (R. Aboughazi [A]). The subcrossed simplicial groups of $H_{*}$ are $\{1\}, C_{*}, S_{*},\{\mathbf{Z} / 2\}, D_{*}, \mathbf{Z} / 2 \times S_{*}$ and $H_{*}$ itself.

Sketch of the proof. First, if $G_{*} \subset S_{*}$ contains a nontrivial permutation then we certainly have $G_{1}=\mathbf{Z} / 2=S_{2}$. Then by looking at $\sigma_{i}^{*}$ we see that $G_{*}$ contains $C_{*}$.

Second, if $G_{*}$ contains a permutation which is not in $C_{*}$ then certainly it contains $(01)$ in $G_{2} \subset S_{3}$. Then it contains (01) in $G_{n} \subset S_{n+1}$ for any $n$. As $S_{n}$ is generated by $(01)$ and $(01 \ldots n)$ it follows that $G_{n}$ contains $S_{n+1}$. 
For $H_{*}$ we use the result for $S_{*}$ and a similar argument for $(\mathbf{Z} / 2)^{n+1}$ where the role of the cyclic permutation is played by $\zeta_{n+1}$ and the role of the permutation $(01)$ in $S_{3}$ is played by the trivial permutation in $S_{2}$ with labels 1 and $y$.

Theorem 3.6 (Classification of crossed simplicial groups). For any crossed simplicial group $G_{*}$ there exists an exact sequence (unique up to isomorphism) of crossed simplicial groups

$$
1 \rightarrow G_{*}^{\prime} \rightarrow G_{*} \rightarrow G_{*}^{\prime \prime} \rightarrow 1
$$

such that $G_{*}^{\prime}$ is a simplicial group and $G_{*}^{\prime \prime}$ is one of the following crossed simplicial groups: $\{1\}, C_{*}, S_{*},\{\mathrm{Z} / 2\}, D_{*}, \mathrm{Z} / 2 \times S_{*}$ and $H_{*}$.

Proof. First we prove that $G_{*}$ determines a unique homomorphism $G_{*} \rightarrow$ $H_{*}$. The image of $G_{n}$ in $S_{n+1}$ has already been determined in 1.7. We now determine the $(\mathbf{Z} / 2)^{n+1}$ part.

Let $g \in G_{n}$ and consider the action of $s_{i}(g)^{-1}$ on the subset $\{i, i+1\}$. If $s_{i}(g)^{-1}$ respects (resp. inverts) the order, then the label of the edge of $g$ abutting at $i$ is 1 (resp. $y$ ).

Hence the map $G_{n} \rightarrow H_{n+1}$ is well defined and this gives a morphism of crossed simplicial groups (see [A] for details).

By definition kernel $G_{*}^{\prime}$ is such that $g \in G_{n}^{\prime}$ acts trivially on [n]. Therefore $g^{*}(\phi)=\phi$ for any $\phi \in \mathrm{Hom}_{\Delta}$. This proves that $G_{*}^{\prime}$ is a simplicial group (cf. Remark 1.8). Finally the theorem follows from Proposition 3.5.

For example, the exact sequence associated to $Q_{*}$ (Example 5 in 1.5) is

$$
1 \rightarrow \mathbf{Z} / 2 \rightarrow Q_{*} \rightarrow D_{*} \rightarrow 1 \text {. }
$$

3.7. The example of braid groups. Let $B_{n}$ be the braid groups on $n$ braids. We denote by $\mu: B_{n} \rightarrow S_{n}$ the canonical surjection and we denote by $T_{n}$ the coloured braid group $\operatorname{Ker} \mu$. Let $\phi$ be in $\operatorname{Hom}_{\Delta}([m],[n])$ and let $g$ be in $B_{n+1}$. We put $g^{*}(\phi)=\mu(g)^{*}(\phi)$, using the definition for the symmetric group.

An analogue of Lemma 3.2 with $B_{n+1}$ in place of $H_{n+1}$ can easily be proved. Hint: use the following picture.

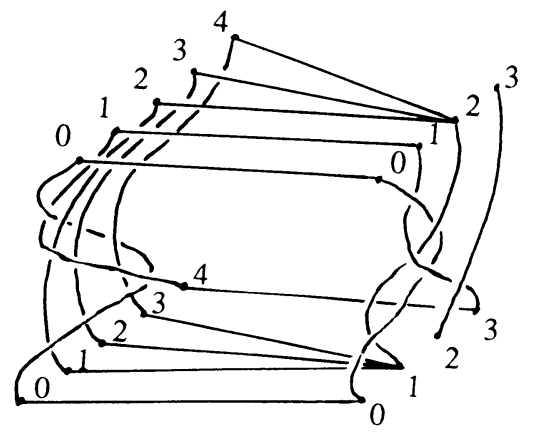


Explicitly the order preserving condition about the restriction of $\phi^{*}(g)$ on $g^{*}(\phi)^{-1}(i)$ is replaced by:

- the restriction of $\phi^{*}(g)$ considered as a braid from $g^{*}(\phi)^{-1}(i)$ to $\phi^{-1}(g(i))$ is the identity braid. The same argument as in the case of the symmetric (and hyperoctahedral) group proves the following.

Theorem 3.8. The family of braid groups $\left\{B_{n+1}\right\}_{n \geq 0}$ is a crossed simplicial group $B_{*}$.

The extension given by the classification theorem is

$$
1 \rightarrow T_{*} \rightarrow B_{*} \rightarrow S_{*} \rightarrow 1,
$$

where $T_{*}$ is a simplicial group.

Remark 3.9. The inverse image of $C_{*} \subset S_{*}$ in $B_{*}$ is the duplicial crossed simplicial group $\mathbf{Z}_{*}$ described in Example 3 of 1.5. The extension provided by 3.6 is

$$
1 \rightarrow \mathbf{Z} \rightarrow \mathbf{Z}_{*} \rightarrow C_{*} \rightarrow 1
$$

where $\mathbf{Z}$ stands for the trivial simplicial group $\mathbf{Z}$. The geometric realization of this extension is the fibration $\mathbf{Z} \rightarrow \mathbf{R} \rightarrow S^{1}$.

Let $K$ be a group and $S_{n+1} \int K=K^{n+1} \rtimes S_{n+1}$ be the wreath product of $S_{n+1}$ by $K$.

Theorem 3.10. The family of groups $\left\{S_{n+1} \int K\right\}_{n \geq 0}$, is a crossed simplicial group $\left(S \int K\right)_{*}$.

Proof. Let $\mu: S_{n+1} \int K \rightarrow S_{n+1}$ be the natural projection. We put $g^{*}(\phi)=$ $\mu(g)^{*}(\phi)$. To define $\phi^{*}(g)$ we consider the geometric definition of $\phi^{*}(g)$ in the symmetric case. An element in $S_{n+1} \int K$ is given by $\left\{\left(k_{0}, \ldots, k_{n+1}\right), g\right\}$ with $k_{i} \in K$ and $g \in S_{n+1}$. We label the vertex going from $i$ to $g(i)$ by $k_{i}$. Therefore the braids of the permutation $\phi^{*}(g)$ are also labelled, and thus it defines an element in $S_{n+1} \int K$ (still denoted $\phi^{*}(g)$ ). The rest of the proof is as in the hyperoctahedral group case.

Remark 3.11. The kernel of the map $\left(S \int K\right)_{*} \rightarrow S_{*}$ is the simplicial group $W(K)$. The simplicial structure of $\left(S \int \mathbf{Z} / 2\right)$ * is different from the simplicial structure of $H_{*}$.

\section{OBJECTS OVER CROSSED SIMPLICIAL GROUPS}

The main justification for studying the simplicial category $\Delta$ is to give a succinct interpretation of the notion of a simplicial object in a category $\mathscr{C}$, that is a functor $\Delta^{\mathbf{o p}} \rightarrow \mathscr{C}$. In this section we define the analogous notion for crossed simplicial groups.

4.1. Definition. Let $G_{*}$ be a crossed simplicial group, let $\Delta G$ be its associated category and let $\mathscr{C}$ be any category. By a $G_{*}$-object lin $\mathscr{C}$ we shall mean a functor $X:(\Delta G)^{\mathbf{o p}} \rightarrow \mathscr{C}$. As in the case of simplicial objects we will denote such 
a functor simply as $X_{*}$ with $X_{n}$ denoting the image of [n]. If $\lambda:[m] \rightarrow[n]$ is a morphism in $\Delta G$ we will denote by $\lambda^{*}: X_{n} \rightarrow X_{m}$ the associated morphism $X(\lambda)$. If $g \in G_{n}$, then noting that $\operatorname{Aut}_{\Delta G}([n])=G_{n}^{\text {op }}$ we will denote by $x \mapsto$ $g x$ (instead of $g^{*} x$ ) the associated action on $X_{n}$ and we will abusively write $G_{n} \times X_{n} \rightarrow X_{n}$. By a morphism of $G_{*}$-objects (or simply $G_{*}$-map) we will mean a natural transformation between functors $(\Delta G)^{\mathbf{o p}} \rightarrow \mathscr{C}$ and we will use the notation $f_{*}: X_{*} \rightarrow Y_{*}$ and $f_{n}: X_{n} \rightarrow Y_{n}$.

In most of our considerations below we will take $\mathscr{C}$ to be either the category of sets or of topological spaces and will refer to the corresponding $G_{*}$-objects as $G_{*}$-sets or $G_{*}$-spaces with the former case being subsumed within the latter by regarding a set as a space with the discrete topology. In $\S 6$ we will consider the case when $\mathscr{C}$ is a category of modules and we will then speak of $G_{*}$-modules.

In the case when $\Delta G=\Delta C$, the cyclic category, our definition reduces to Connes' notion of a cyclic object. As in that particular case, it is useful to give another interpretation of a $G_{*}$-object as a simplicial object with some additional structure.

Lemma 4.2. The notion of a $G_{*}$-object in $\mathscr{C}$ is equivalent to the notion of a simplicial object $X_{*}$ in $\mathscr{C}$ with the following additional structure:

(a) left group actions $G_{n} \times X_{n} \rightarrow X_{n}$,

(b) face relations (cf. 1.7) $d_{i}(g x)=d_{i}(g)\left(d_{g^{-1} i} x\right)$,

(c) degeneracy relations (cf. 1.7) $s_{i}(g x)=s_{i}(g)\left(s_{g^{-1}(i)} x\right)$.

In fact it suffices to specify the face and degeneracy relations for the generators of $G_{n}$. A $G_{*}$-map $f_{*}: X_{*} \rightarrow Y_{*}$ is the same thing as a simplicial map such that each $f_{n}: X_{n} \rightarrow Y_{n}$ is $G_{n}$-equivariant.

Proof. The inclusion $\Delta \subseteq \Delta G$ defines for each $G_{*}$-object $X_{*}$ an underlying simplicial object which we also denote $X_{*}$. The stated equivalence follows readily from the characterization of crossed simplicial groups given in Proposition 1.7 .

It is now easy to define a free functor from simplicial spaces to $G_{*}$-spaces which is adjoint to the forgetful functor going the other way.

Definition 4.3. Let $X_{*}$ be a simplicial space. Define a $G_{*}$-space $F_{G_{*}}\left(X_{*}\right)$ whose space of $n$-simplices is $G_{n} \times X_{n}$ with $G_{n}$-action specified by left multiplication on $G_{n}$ and with faces and degeneracies specified by the formulas

$$
\begin{aligned}
& d_{i}(g, x)=\left(d_{i}(g), d_{g^{-1}(i)}(x)\right), \\
& s_{i}(g, x)=\left(s_{i}(g), s_{g^{-1}(i)}(x)\right) .
\end{aligned}
$$

We define a simplicial map $l_{*}: X_{*} \rightarrow F_{G_{*}}\left(X_{*}\right)$ by $x \rightarrow(1, x)$ and a $G_{*}$-map $\mu_{*}: F_{G_{*}} F_{G_{*}}\left(X_{*}\right) \rightarrow F_{G_{*}}\left(X_{*}\right)$ by $\left(g_{1}, g_{2}, \dot{x}\right) \rightarrow\left(g_{1} g_{2}, x\right)$. If $X_{*}$ is a $G_{*}$-space define a $G_{*}$-map $e v_{*} \vdots F_{G_{*}}\left(X_{*}\right) \rightarrow X_{*}$ by $(g, x) \rightarrow g x$.

It is an easy exercise that we leave to the diligent reader to verify that 
Proposition 4.4. (i) $F_{G_{*}}\left(X_{*}\right)$ as specified above is a $G_{*}$-space, (ii) $F_{G_{*}}$ is left adjoint to the forgetful map from $G_{*}$-spaces to simplicial spaces, with adjunction specified by $l_{*}$ and coadjunction specified by ev $v_{*}$, (iii) $\left(F_{G_{*}}, \mu_{*}, l_{*}\right)$ specifies a monad on the category of simplicial spaces whose algebras are precisely the $G_{*}$-spaces.

Exercise 4.5. The $G_{*} n$-simplex $\Delta_{*}^{n}\left(G_{*}\right):(\Delta G)^{\mathrm{op}} \rightarrow$ Sets is the functor specified by $\operatorname{Hom}_{\Delta G}(-,[n])$. Show that $\Delta_{*}^{n}\left(G_{*}\right)=F_{G_{*}}\left(\Delta_{*}^{n}\right)$ where $\Delta_{*}^{n}$ is the $n$-simplex.

\section{GeOMETRIC REALIZATIONS OF CROSSED SIMPLiCIAL GROUPS}

When A. Connes defined the cyclic category $\Delta C$ and the notion of a cyclic object, he showed that in some sense he was codifying combinatorially the notion of an action of the circle group $S^{1}$ and he showed that the classifying space $B \Delta C$ was equivalent to the classifying space of the circle $B S^{1}=\mathbf{C} P^{\infty}$. In this section we extend Connes' results to arbitrary crossed simplicial groups and strengthen them even for the cyclic case. We show that for any crossed simplicial group $G_{*}$, the geometric realization of the underlying simplicial set is a topological group $\left|G_{*}\right|$, that the geometric realization of a $G_{*}$-space is a $\left|G_{*}\right|$-space, and that the homotopy colimit of a $G_{*}$-space $X_{*}$ regarded as a functor $\Delta G^{\mathbf{0 p}} \rightarrow$ Top is equivalent to the Borel construction $E\left|G_{*}\right| \times_{\left|G_{*}\right|}\left|X_{*}\right|$. Finally we prove a theorem which severely restricts the kinds of topological groups which can arise in this way.

Proposition 5.1. $G_{*}$ be a crossed simplicial group and $X_{*}$ be a simplicial space. Then there are projection maps $p_{1}:\left|F_{G_{*}}\left(X_{*}\right)\right| \rightarrow\left|G_{*}\right|$ and $p_{2}:\left|F_{G_{*}}\left(X_{*}\right)\right| \rightarrow\left|X_{*}\right|$ having the following properties:

(i) $\left(p_{1}, p_{2}\right):\left|F_{G_{*}}\left(X_{*}\right)\right| \rightarrow\left|G_{*}\right| \times\left|X_{*}\right|$ is a homeomorphism.

(ii) For any simplicial map $f_{*}: X_{*} \rightarrow Y_{*}$ the following diagrams commute:

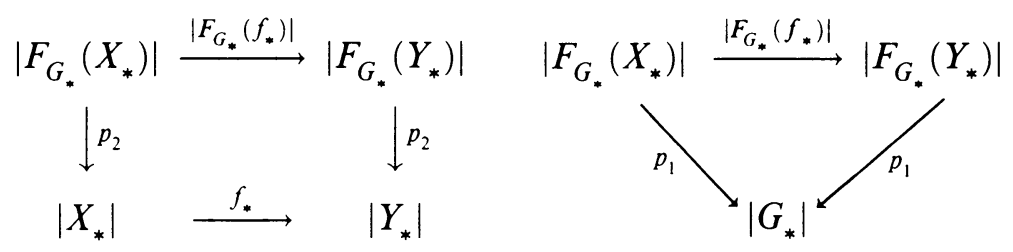

(iii) $p_{2}:\left|F_{G_{*}}\left(X_{*}\right)\right| \rightarrow\left|X_{*}\right|$ defines an action of the monad $\left(F_{G_{*}}, \mu_{*}, l_{*}\right)$ on the geometric realization functor; i.e. the following diagrams commute:

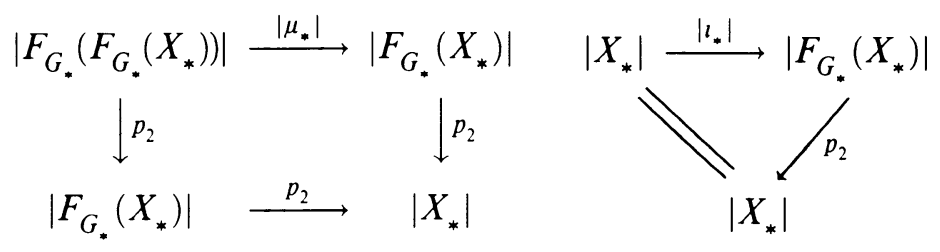

(iv) The composite $\left|G_{*}\right| \cong\left|F_{G_{*}}(*)\right| \stackrel{p_{1}}{\longrightarrow}\left|G_{*}\right|$ is the identity. 
(v) The following diagram commutes:

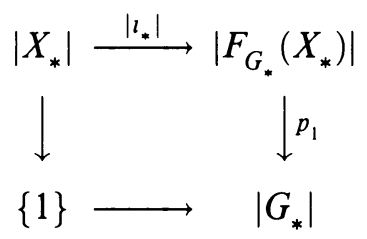

To prove this we first need the following lemma.

Lemma 5.2. Let $G_{*}$ be a crossed simplicial group. Then there are affine actions $G_{n} \times \Delta^{n} \rightarrow \Delta^{n}$ such that for any morphism $\phi:[m] \rightarrow[n]$ in $\Delta$ and any $g \in G_{n}$ the following diagram commutes:

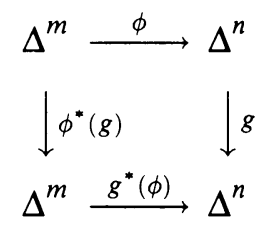

Proof. The defining representations $G_{n} \rightarrow S_{n+1}$ of a crossed simplicial group extend obviously to affine actions $G_{n} \times \Delta^{n} \rightarrow \Delta^{n}$. The result now follows from Proposition 1.7.

Proof of Proposition 5.1. There is a simplicial projection $p_{1 *}: F_{G_{*}}\left(X_{*}\right) \rightarrow G_{*}$ given by $p_{1 *}(g, x)=g$. We take $p_{1}=\left|p_{1 *}\right|$. We define $p_{2}:\left|F_{G_{*}}\left(X_{*}\right)\right| \rightarrow\left|X_{*}\right|$ by the following prescription:

$$
p_{2}[(g, x), u]=[x, g \cdot u] .
$$

Here $u \in \Delta^{n}$ and $g \cdot u$ refers to the action constructed in Lemma 5.2. The following calculation shows that $p_{2}$ is well defined:

$$
\begin{aligned}
p_{2}\left[\left(\phi^{*}(g, x), u\right)\right] & =p_{2}\left[\left(\phi^{*}(g),\left(g^{*}(\phi)\right)^{*}(x)\right), u\right] \\
& =\left[\left(g^{*}(\phi)^{*}(x), \phi^{*}(g) u\right]=\left[x, g^{*}(\phi) \cdot \phi^{*}(g) u\right]\right. \\
& =[x, g \cdot \phi(u))=p_{2}((g, x), \phi(u)]
\end{aligned}
$$

where $g \in G_{n}, x \in X_{n} \quad u \in \Delta^{m}$ and $\phi:[m] \rightarrow[n]$ is in $\Delta$.

It is straightforward to check that properties (ii) $-(\mathrm{v})$ hold. To prove (i) we first construct a homeomorphism $h:\left|G_{*} \times X_{*}\right| \rightarrow\left|F_{G_{*}}\left(X_{*}\right)\right|$ by defining

$$
h[(g, x), u]=\left[\left(g^{-1}, x\right), g \cdot u\right]
$$

A similar calculation as above shows that $h$ is well defined, and it is obviously a homeomorphism. In the case when $X_{*}$ is a point we get a homeomorphism $l:\left|G_{*}\right| \rightarrow\left|G_{*}\right|$ given by

$$
l[g, u]=\left[g^{-1}, g \cdot u\right]
$$


We deduce (i) by noting that the following diagram commutes:

$$
\begin{array}{rr}
\left|G_{*} \times X_{*}\right| \stackrel{h}{\longrightarrow}\left|F_{G_{*}}\left(X_{*}\right)\right| \\
\downarrow \downarrow \mid\left(\left|q_{1 *}\right|,\left|q_{2 *}\right|\right) & \downarrow\left(p_{1}, p_{2}\right) \\
\left|G_{*}\right| \times\left|X_{*}\right| \stackrel{\imath \times \text { id }}{\longrightarrow}\left|G_{*}\right| \times\left|X_{*}\right|
\end{array}
$$

where $q_{1 *}: G_{*} \times X_{*} \rightarrow G_{*}$ and $q_{2 *}: G_{*} \times X_{*} \rightarrow X_{*}$ are the standard simplicial projections.

Theorem 5.3. (i) If $G_{*}$ is a crossed simplicial group, then $\left|G_{*}\right|$ is a topological group.

(ii) If $X_{*}$ is a $G_{*}$-space, then there is an induced action $\left|G_{*}\right| \times\left|X_{*}\right| \rightarrow\left|X_{*}\right|$.

(iii) $\left(p_{1}, p_{2}\right):\left|F_{G_{*}}\left(X_{*}\right)\right| \rightarrow\left|G_{*}\right| \times\left|X_{*}\right|$ is an equivariant homeomorphism.

Proof. If $X_{*}$ is a $G_{*}$-space, then there is an evaluation map $e v_{*}: F_{G_{*}}\left(X_{*}\right) \rightarrow$ $X_{*}$, and we define a map $\zeta:\left|G_{*}\right| \times\left|X_{*}\right| \rightarrow\left|X_{*}\right|$ as the following composite:

$$
\left|G_{*}\right| \times\left|X_{*}\right| \stackrel{\left(p_{1}, p_{2}\right)^{-1}}{\longrightarrow}\left|F_{G_{*}}\left(X_{*}\right)\right| \stackrel{\left|e v_{*}\right|}{\longrightarrow}\left|X_{*}\right|
$$

In particular $\left|G_{*}\right|=\left|F_{G_{*}}\left(G_{*}\right)\right|$ is a $G_{*}$-space (via $\mu_{*}$ ), and so we get a map $\zeta^{G_{*}}:\left|G_{*}\right| \times\left|G_{*}\right| \rightarrow\left|G_{*}\right|$. We first note that for any simplicial space $X_{*}$ we have the following commutative diagram:

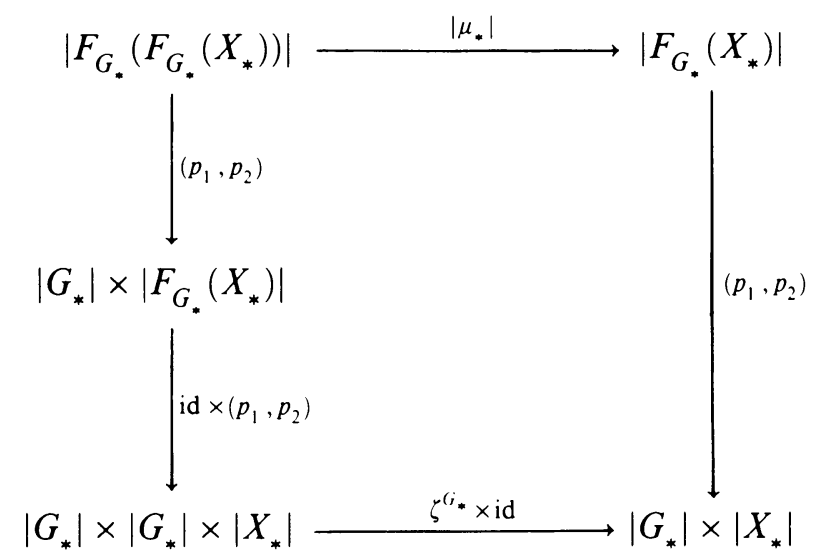

This follows from parts (ii), (iii) and (iv) of Proposition 5.1, the naturality of $\mu_{*}$, and the fact that for $X_{*}=*$ the diagram commutes by definition of $\zeta^{G}$. 
On the other hand the diagram

$$
\begin{aligned}
& \left|F_{G_{*}}\left(F_{G_{*}}\left(X_{*}\right)\right)\right| \stackrel{\left|F_{G_{*}}\left(\mathrm{ev}_{*}\right)\right|}{\longrightarrow}\left|F_{G_{*}}\left(X_{*}\right)\right| \stackrel{\mid \mathrm{ev}}{\longrightarrow}\left|X_{*}\right| \\
& \downarrow\left(p_{1}, p_{2}\right) \quad \downarrow\left(p_{1}, p_{2}\right) \quad \| \\
& \left|G_{*}\right| \times\left|F_{G_{*}}\left(X_{*}\right)\right| \stackrel{\mathrm{id} \times\left|\mathrm{ev}_{*}\right|}{\longrightarrow}\left|G_{*}\right| \times\left|X_{*}\right| \stackrel{\zeta}{\longrightarrow}\left|X_{*}\right| \\
& \downarrow \text { id } \times\left(p_{1}, p_{2}\right) \quad \| \\
& \left|G_{*}\right| \times\left|G_{*}\right| \times\left|X_{*}\right| \stackrel{\text { id } \times \zeta}{\longrightarrow}\left|G_{*}\right| \times\left|X_{*}\right| \stackrel{\zeta}{\longrightarrow}\left|X_{*}\right|
\end{aligned}
$$

commutes by part (ii) of Proposition 5.1 and the definition of $\zeta$. It then follows from the associativity of the monad action of $F_{G_{*}}$ on $X_{*}$ that

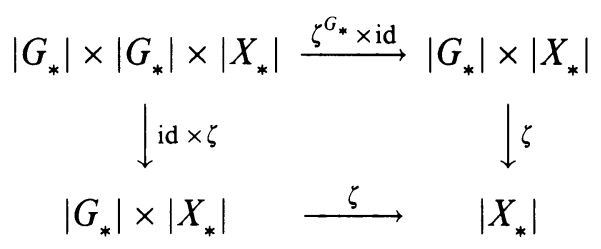

commutes. In particular taking $X_{*}=G_{*}$ we obtain that $\zeta^{G_{*}}:\left|G_{*}\right| \times\left|G_{*}\right| \rightarrow\left|G_{*}\right|$ is an associative multiplication.

Now it follows from part (iii) of Proposition 5.1 that

$$
\begin{aligned}
& \left|X_{*}\right| \stackrel{\left|u_{*}\right|}{\longrightarrow}\left|F_{G_{*}}\left(X_{*}\right)\right| \\
& \downarrow \cong \quad \downarrow\left(p_{1}, p_{2}\right) \\
& \{1\} \times\left|X_{*}\right| \longrightarrow\left|G_{*}\right| \times\left|X_{*}\right|
\end{aligned}
$$

commutes, which together with the unit diagram for the action of the monad $F_{G_{*}}$ on $X_{*}$ implies that

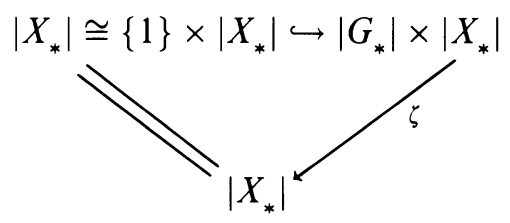

commutes. In particular, taking $X_{*}=G_{*}$ we obtain that $1 \in\left|G_{*}\right|$ is a left unit. By part (ii) of Proposition 5.1 and the definition of $\zeta^{G}$ the following diagram also commutes:

$$
\begin{aligned}
& \left|G_{*}\right| \cong\left|F_{G_{*}}(*)\right| \hookrightarrow\left|F_{G_{*}}\left(G_{*}\right)\right| \stackrel{\left|e v_{*}\right|}{\longrightarrow}\left|G_{*}\right| \\
& \left\|\quad \downarrow\left(p_{1}, p_{2}\right) \quad\right\| \\
& \left|G_{*}\right| \cong\left|G_{*}\right| \times\left|G_{*}\right|\{1\} \hookrightarrow\left|G_{*}\right| \times\left|G_{*}\right| \stackrel{\zeta^{(j}}{\longrightarrow}\left|G_{*}\right|
\end{aligned}
$$


and by inspection the composite across the top is the identity. Hence $1 \in\left|G_{*}\right|$ is also a right unit.

We also claim that the homeomorphism $l:\left|G_{*}\right| \rightarrow\left|G_{*}\right|$ constructed in the proof of Proposition 5.1 is the inverse. To see this, note that according to the proof of Proposition 5.1 and the definition of $\zeta^{G}$, the following diagram commutes:

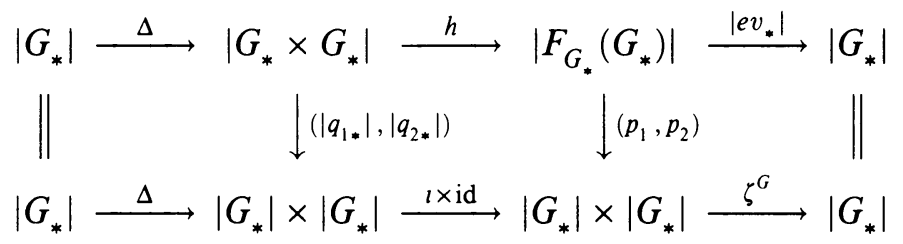

and by inspection the composite across the top is the constant map at 1 . Hence $l$ is a left inverse. The fact that $l^{2}=$ id implies that $l$ is also a left inverse.

Part (iii) follows readily from the constructions above. This completes the proof.

The following fundamental example illustrates the above results.

Example 5.4. Let $G_{*}=C_{*}$. Then the free cyclic set $F_{C_{*}}\left(S_{*}^{1}\right)$ corresponds to the following triangulation of the torus

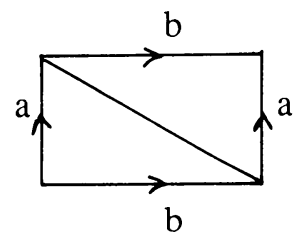

as opposed to

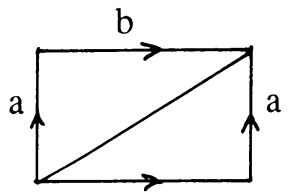

b

which corresponds to $S_{*}^{1} \times S_{*}^{1}$, the standard simplicial product. The multiplication map $S_{*}^{1} \times S_{*}^{1}=I^{2} / \sim \rightarrow S^{1}$ given by

$$
(s, t) \rightarrow \begin{cases}s+t & \text { if } s+t \leq 1 \\ s+t-1 & \text { if } s+t \geq 1\end{cases}
$$

is simplicial with respect to the first triangulation (corresponding to ev $: F_{C_{*}}\left(S_{*}^{1}\right)$ $\rightarrow S_{*}^{1}$ ) but is not simplicial with respect to the second triangulation.

The following example illustrates the kind of group actions which can be codified in the context of crossed simplicial groups.

Example 5.5. The action of $S^{1}$ on $S^{3}$ given by quaternionic multiplication can be realized as a geometric realization of a cyclic set. One first notes that $S^{3}$ with this $S^{1}$ action is equivariantly homeomorphic to the join $S^{1} * S^{1}$ with $S^{1}$ acting on both factors of the join. This in turn is the realization of the cyclic 
set $X_{*}$ obtained by taking the pushout diagram

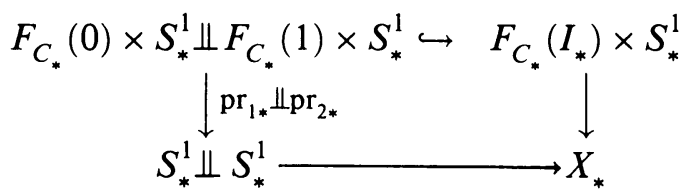

where $\operatorname{pr}_{1 *}$ denotes projection onto the first factor $F_{C_{*}}(0)=S_{*}^{1}$ and $\operatorname{pr}_{2 *}$ denotes projection into the second factor.

Several of our subsequent results will require an explicit formula for the action of $\left|G_{*}\right|$ on the geometric realization $\left|X_{*}\right|$ of a $G_{*}$-space. Such a formula is furnished by the following lemma.

Lemma 5.6. Let $G_{*}$ be a crossed simplicial group and let $X_{*}$ be a $G_{*}$-space. Then

(i) For any $g \in G_{m},\left(s_{0}, s_{1}, \ldots, s_{m}\right) \in \Delta^{m},\left(t_{0}, t_{1}, \ldots, t_{n}\right) \in \Delta^{n}$ there are surjections $\lambda:[p] \rightarrow[m], \gamma:[p] \rightarrow[n]$ in $\Delta$ and an element $\left(u_{0}, u_{1}, \ldots, u_{p}\right) \in$ $\Delta$ such that $\lambda\left(u_{0}, u_{1}, \ldots, u_{p}\right)=\left(s_{0}, s_{1}, \ldots, s_{m}\right)$ and $\gamma \lambda^{*}(g)\left(u_{0}, u_{1}, \ldots, u_{p}\right)$ $=\left(t_{0}, t_{1}, \ldots, t_{n}\right)$.

(ii) For any $g \in G_{n}, x \in X_{n}$ and $\left(t_{0}, t_{1}, \ldots, t_{n}\right) \in \Delta^{n}$

$$
\left[g,\left(t_{0}, t_{1}, \ldots, t_{n}\right)\right] \cdot\left[x, g\left(t_{0}, t_{1}, \ldots, t_{n}\right)\right]=\left[g x,\left(t_{0}, t_{1}, \ldots, t_{n}\right)\right] .
$$

Proof. By the shuffle algorithm we can find surjections $\alpha:[p] \rightarrow[m], \gamma:[p] \rightarrow$ $[n]$ in $\Delta$ and an element $\left(v_{0}, v_{1}, \ldots, v_{p}\right) \in \Delta^{p}$ such that $\alpha\left(v_{0}, v_{1}, \ldots, v_{p}\right)=$ $g\left(s_{0}, s_{1}, \ldots, s_{m}\right)$ and $\gamma\left(v_{0}, v_{1}, \ldots, v_{p}\right)=\left(t_{0}, t_{1}, \ldots, t_{n}\right)$. Let $\lambda=\left(g^{-1}\right)^{*}(\alpha)$. It then follows from Lemma 5.2 that

$$
\lambda \cdot \lambda^{*}(g)^{-1}\left(v_{0}, v_{1}, \ldots, v_{p}\right)=\left(s_{0}, s_{1}, \ldots, s_{m}\right) .
$$

If we let $\left(u_{0}, u_{1}, \ldots, u_{p}\right)=\lambda^{*}(g)^{-1}\left(v_{0}, v_{1}, \ldots, v_{p}\right)$, part (i) follows immediately. Part (ii) follows from noting that

$$
\begin{aligned}
& \left(p_{1}, p_{2}\right)^{-1}\left(\left[g,\left(t_{0}, t_{1}, \ldots, t_{n}\right)\right],\left[x, g\left(t_{0}, t_{1}, \ldots, t_{n}\right)\right]\right) \\
& \quad=\left[(g, x),\left(t_{0}, t_{1}, \ldots, t_{n}\right)\right] .
\end{aligned}
$$

Theorem 5.7. Let $G_{*}$ be a crossed simplicial group and denote by $\left|G_{*}\right|$-Top, resp. $G_{*}$-Sets, the categories of $\left|G_{*}\right|$-spaces and $G_{*}$-sets respectively. Then || . $G_{*}$-Sets $\rightarrow\left|G_{*}\right|$-Top has a right adjoint Sing $_{*}:\left|G_{*}\right|-$ Top $\rightarrow G_{*}$-Sets obtained by endowing the total singular complex of $a\left|G_{*}\right|$-space with the structure of $a G_{*}$-set. Proof. Let $X$ be a $\left|G_{*}\right|$-space, let $\sigma: \Delta^{n} \rightarrow X$ be a singular simplex, and let $g \in G_{n}$. Define the singular simplex $g \cdot \sigma: \Delta^{n} \rightarrow X$ by the following formula

$$
(g \cdot \sigma)(u)=[g, u] \cdot \sigma(g u)
$$

for $u \in \Delta^{n}$. It follows easily from Lemmas 5.2 and 5.6 that this endows the total singular complex $\operatorname{Sing}_{*}\left(X_{*}\right)$ with the structure of a $G_{*}$-set. Now apply the standard adjunction between topological spaces and simplicial sets, noting (by 
Lemma 5.6) that for a $G_{*}$-set $Y_{*}$ the unit map $Y_{*} \rightarrow \operatorname{Sing}_{*}\left(\left|Y_{*}\right|\right)$ is a $G_{*}$-map, and that for a $\left|G_{*}\right|$-space $X$ the counit map $\left|\operatorname{Sing}_{*}(X)\right| \rightarrow X$ is a $\left|G_{*}\right|$-map.

Remark. In the paragraph below we prove various results about homotopy colimits over the category $\Delta G$. For the convenience of the reader we first recall the definition of hocolim $X$ where $X$ is a functor from a small category $\mathscr{C}$ to Sets or Top. Let $\mathscr{C}_{X}$ be the category whose objects are $(C, x)$ where $C$ is an object of $\mathscr{C}$ and $x \in X(C)$. A morphism $(C, x)$ to $\left(C^{\prime}, x^{\prime}\right)$ in $\mathscr{C}_{X}$ is a morphism $C \rightarrow C^{\prime}$ in $\mathscr{C}$ such that $f_{*}(x)=x^{\prime}$. The nerve of this category is a simplicial set whose geometric realization is, by definition, hocolim $X$ (cf. [B-K]).

Proposition 5.8. $\Omega B \Delta G \cong\left|G_{*}\right|$.

Proof. Consider the inclusion functor $i: \Delta \rightarrow \Delta G$ and apply Quillen's Theorem B [Q]. For any object $[m]$ of $\Delta G$ consider the category $[m] \backslash i$. This has as objects all arrows of the form $f:[n] \rightarrow[m]$ in $\Delta G$ and as morphisms all commutative diagrams of the form

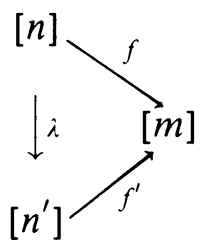

in $\Delta G$ with $\lambda$ required to be in $\Delta$. We can identify the classifying space $B([m] \backslash i)$ with the homotopy colimit of the functor $\operatorname{Hom}_{\Delta G}(-,[m]): \Delta^{\mathbf{o p}} \rightarrow$ Sets . But the homotopy colimit of a simplicial set is naturally homotopy equivalent to its geometric realization. Thus it follows from Exercise 4.5 that $B([m] \backslash i)$ is equivalent to $\left|F_{G_{*}}\left(\Delta^{m}\right)\right| \cong\left|G_{*}\right| \times\left|\Delta^{m}\right|$. By naturality of this equivalence any morphism $\gamma:[m] \rightarrow\left[m^{\prime}\right]$ in $\Delta$ induces an equivalence $B([m] \backslash i) \rightarrow B\left(\left[m^{\prime}\right] \backslash i\right)$, and since any morphism in $\Delta G$ is a composite of a morphism in $\Delta$ and an isomorphism, the same must be true for an arbitrary morphism $f:[m] \rightarrow\left[\mathrm{m}^{\prime}\right]$ in $\Delta G$. Quillen's Theorem B allows us to conclude that we have a fibration sequence $\left|G_{*}\right| \rightarrow B \Delta \rightarrow B \Delta G$. But $\Delta$ has a terminal object [0], so $B \Delta$ is contractible. Hence $\Omega B \Delta G \simeq\left|G_{*}\right|$.

Theorem 5.9. For any $G_{*}$-space $X_{*}$ there is a natural fibration sequence

$$
\left|X_{*}\right| \rightarrow \text { hocolim }_{\Delta G} X_{*} \rightarrow B \Delta G \text {. }
$$

Proof. We first prove the theorem for $G_{*}$-sets. Here $\mathscr{C}=\Delta G^{\text {op }}$ and we denote by $X_{\Delta}$ the restriction of $X$ to $\Delta^{\mathbf{o p}}$. Let $i_{X}: X_{\Delta} \rightarrow X$ denote the inclusion functor. Now it is easy to see that for any object $x$ of $X$ the category $x / i_{X}$ is isomorphic to the opposite of the category $[\mathrm{m}] \backslash i$ considered in the preceding paragraph. Again by Quillen's Theorem B we obtain a natural fibration sequence

$$
\left|G_{*}\right| \rightarrow\left|X_{*}\right| \rightarrow \text { hocolim }_{\Delta G} X_{*} .
$$


Comparing this with the case of a trivial $G_{*}$-space, we get a map of fibration sequences

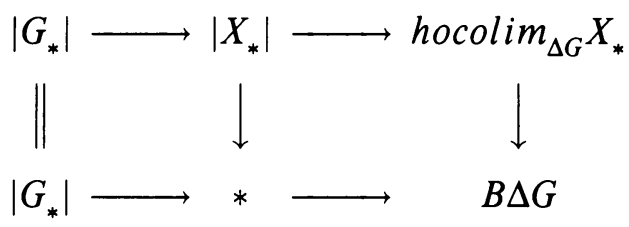

We conclude

$$
\left|X_{*}\right| \rightarrow \text { hocolim }_{\Delta G} X_{*} \rightarrow B \Delta G
$$

is a fibration.

For the general (nondiscrete) case, we replace $X_{*}: \Delta G^{\mathbf{0 p}} \rightarrow$ Top by the composite

$$
\Delta G^{\mathrm{op}} \stackrel{X_{*}}{\longrightarrow} \text { Top } \stackrel{\text { Sing }_{*}}{\longrightarrow} \text { Simplicial sets }
$$

and apply the following well-known result: if $A_{* *} \rightarrow B_{* *} \rightarrow C_{* *}$ is a sequence of bisimplicial sets such that for each $n,\left|C_{* n}\right|$ is connected and $A_{* n} \rightarrow B_{* n} \rightarrow C_{* n}$ is a fibration sequence, then so is $\left|A_{* *}\right| \rightarrow\left|B_{* *}\right| \rightarrow\left|C_{* *}\right|$.

Corollary 5.10. If $f_{*}: X_{*} \rightarrow Y_{*}$ is a map of $G_{*}$-spaces such that $\left|f_{*}\right|:\left|X_{*}\right| \rightarrow\left|Y_{*}\right|$ is an equivalence, then so is

$$
\operatorname{hocolimf}_{*}: \operatorname{hocolim}_{\Delta G} X_{*} \rightarrow \text { hocolim }_{\Delta G} Y_{*} .
$$

Proposition 5.11. (i) For any simplicial space $X_{*}$ there is a natural equivalence

$$
d: \operatorname{hocolim}_{\Delta G} F_{G}\left(X_{*}\right) \rightarrow\left|X_{*}\right| \text {. }
$$

(ii) The following diagram commutes:

$$
\begin{aligned}
& \operatorname{hocolim}_{\Delta G} F_{G}\left(F_{G}\left(X_{*}\right)\right) \stackrel{\delta}{\longrightarrow}\left|F_{G}\left(X_{*}\right)\right| \\
& \downarrow \operatorname{hocolim} \mu \quad \downarrow p_{2} \\
& \operatorname{hocolim}_{\Delta G} F_{G}\left(X_{*}\right) \stackrel{\delta}{\longrightarrow}\left|X_{*}\right| \text {. }
\end{aligned}
$$

Proof. The proof is basically an elaboration of the proof of Proposition 1.11 given in [B-K]. We first identify the homotopy colimit as a coend

$$
\begin{aligned}
\text { hocolim }_{\Delta G} F_{G}\left(X_{*}\right) & =\int_{[m] \in \Delta G} F_{G}\left(X_{*}\right)_{m} \times B(\Delta G /[m]) \\
& =\int_{[m] \in \Delta G} \int_{[n] \in \Delta} X_{n} \times \operatorname{Hom}_{\Delta G}([m],[n]) \times B(\Delta G /[m]) \\
& =\int_{[n] \in \Delta} X_{n} \times \int_{[m] \times \Delta G} \operatorname{Hom}_{\Delta G}([m],[n]) \times B(\Delta G /[m]) \\
& =\int_{[n] \in \Delta} X_{n} \times B(\Delta G /[n]) .
\end{aligned}
$$


We now construct a cosimplicial equivalence $d_{n}: B(\Delta G /[n]) \rightarrow \Delta^{n}$ as follows: given an element

$$
P=\left[\stackrel{\left[m_{0}\right] \rightarrow\left[m_{1}\right] \rightarrow \ldots \rightarrow\left[m_{k}\right]}{f_{f_{1}} / f_{k}},\left(u_{0}, u_{1}, \ldots, u_{k}\right)\right]
$$

in $B(\Delta G /[n])$, construct the affine map $\rho_{P}: \Delta^{k} \rightarrow \Delta^{n}$ by sending the $j$ th vertex of $\Delta^{k}$ of $f_{j}(1,0, \ldots, 0)$ where $f_{j}: \Delta^{k} \rightarrow \Delta^{n}$ is the affine map defined by using Lemma 5.2. We define $d_{n}(P)=\rho_{P}\left(u_{0}, u_{1}, \ldots, u_{k}\right)$. This is easily checked to be well defined and cosimplicial. It is also an equivalence since $B(\Delta G /[n])$ is contractible as is $\Delta^{n}$ and the equalizer of $d^{0}, d^{1}: B(\Delta G /[0]) \rightarrow B(\Delta G /[1])$ is empty. Hence we have an equivalence $d=\int_{[n] \in \Delta} \mathrm{id} \times d_{n}$ : hocolim $_{\Delta G} F_{G}\left(X_{*}\right)=$ $\int_{[n] \in \Delta} X_{n} \times B(\Delta G /[n]) \rightarrow \int_{[n] \in \Delta} X_{n} \times \Delta^{n}=\left|X_{*}\right|$.

The commutativity of (ii) is a tedious but straightforward exercise.

Theorem 5.12. For any $G_{*}$-space $X_{*}$ there is a natural equivalence of fibration sequences

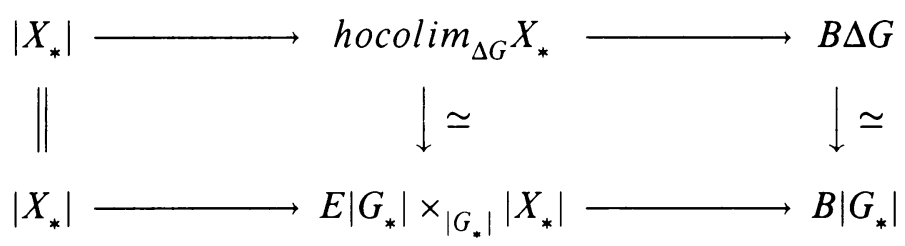

Proof. The map ev $: F_{G}\left(X_{*}\right) \rightarrow X_{*}$ defines a map of simplicial $G_{*}$-spaces $\chi_{*}: B\left(F_{G}, F_{G}, X_{*}\right) \rightarrow X_{*}$, where $B\left(F_{G}, F_{G}, X_{*}\right)$ denotes the monadic twosided bar construction whose space of $k$-simplices is the $(k+1)$-fold iterate

$$
F_{G}\left(F_{G}\left(\ldots F_{G}\left(X_{*}\right) \ldots\right)\right)
$$

with faces given by $e v_{*}$ and the monadic multiplication $\mu_{*}$ and with degeneracies given by the monadic unit $l_{*}$. Moreover, the geometric realization $\left|\chi_{*}\right|$ is an equivalence, so by Corollary $5.10 \chi_{*}$ induces an equivalence between hocolim $_{\Delta G} X_{*}$ and hocolim $\Delta G\left(F_{G}, F_{G}, X_{*}\right)$. But by Proposition 5.11

$$
\begin{aligned}
\text { hocolim }_{\Delta G} B\left(F_{G}, F_{G}, X_{*}\right) & =\mid n \mapsto \text { hocolim }_{\Delta G} B_{n}\left(F_{G}, F_{G}, X_{*}\right) \mid \\
\simeq\left|n \mapsto\left(\left|G_{*}\right|^{n} \times\left|X_{*}\right|\right)\right| & =E\left|G_{*}\right| \times_{\left|G_{*}\right|}\left|X_{*}\right| .
\end{aligned}
$$

In particular for the trivial $G_{*}$-space we obtain $B \Delta G \simeq B\left|G_{*}\right|$. By naturality we obtain the above equivalence of fibration sequences.

Proposition 5.13. (i) If $G_{*}$ is a crossed simplicial group, there is for every $n$ an inclusion of $G_{n} \subset\left|G_{*}\right|$ as a discrete subgroup.

(ii) If $m+1$ divides $n+1$ there is a degeneracy $\eta:[n] \rightarrow[m]$ in $\Delta$ such that $\eta$ induces an inclusion of groups $G_{m} \subset G_{n}$ so that the following diagram 
commutes:

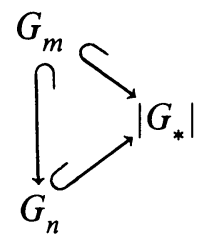

(iii) $\bigcup G_{n}$ is a dense subgroup of $\left|G_{*}\right|$.

Proof. Define $G_{n} \subset\left|G_{*}\right|$ by the specification

$$
g \mapsto[g,(1 /(n+1), 1 /(n+1), \ldots, 1 /(n+1))] .
$$

This is easily seen to be injective with discrete image, and by Lemma 5.6 it is a group homomorphism. The degeneracy $\eta$ specified in (ii) is given by the surjection $[n] \rightarrow[m]$ which maps blocks of successive $k$ elements of $[n]$ into successive elements of $[m]$, where $n+1=k(m+1)$.

For (iii) note that elements of the form $\left[g,\left(u_{0}, u_{1}, \ldots, u_{n}\right)\right]$ with rational barycentric coordinates $u_{i}$ are evidently dense in $\left|G_{*}\right|$. But given such an element $\left[g,\left(u_{0}, u_{1}, \ldots, u_{n}\right)\right]$ we can find a degeneracy $\lambda:[p] \rightarrow[n]$ such that

$$
\lambda(1 /(p+1), 1 /(p+1), \ldots, 1 /(p+1))=\left(u_{0}, u_{1}, \ldots, u_{n}\right) .
$$

Thus $\left[g,\left(u_{0}, u_{1}, \ldots, u_{n}\right)\right]=\left[\lambda^{*}(g),(1 /(p+1), 1 /(p+1), \ldots, 1 /(p+1))\right] \epsilon$ $G_{p}$.

Example 5.14. When $G_{*}=C_{*}$, the inclusion $G_{n}=\mathrm{Z} / n+1 \subset\left|G_{*}\right|=S^{1}$ corresponds to the inclusion of $(n+1)$ th roots of unity.

Theorem 5.15. If $G_{*}$ is a crossed simplicial group such that the geometric realization $\left|G_{*}\right|$ is a Lie group, then the path component of the identity of $\left|G_{*}\right|$ is nilpotent.

Proof. According to a theorem of Zassenhaus, Kazdan and Margulis (cf. [R]), in any Lie group $G$ there is a neighborhood $U$ of the identity such that for any discrete subgroup $\Gamma$ of $G$ the intersection $\Gamma \cap U$ is contained in some nilpotent subgroup of $G$. Take $G=\left|G_{*}\right|$ and let $U$ be the corresponding neighborhood.

Let $N_{n}$ be the minimal nilpotent subgroup of $G$ which contains $U \cap G_{n}$. Since if $m+1$ divides $n+1, G_{m}$ is a subgroup of $G_{n}$, it follows that in this case $N_{m} \subset N_{n}$. One can therefore conclude that $N=\bigcup N_{n}$ is a nilpotent subgroup of $G$ containing $\left(\bigcup G_{n}\right) \cap U$. But $\bigcup G_{n}$ is dense in $G$ so $\left(\bigcup G_{n}\right) \cap U$ is dense in $U$. Hence $U \subset N$. But $U$ is a neighborhood of the identity in $G$, so it follows that $N$ contains the path component of the identity in $G$.

Remark 5.16. By using the classification theorem (Theorem 3.6) we can verify that under the hypothesis of Theorem 5.15, the path component of the identity of $\left|G_{*}\right|$ has to be either $\{1\}, S^{1}$ or $\mathbf{R}$. However, the argument above is more general, applying to multisimplicial generalizations of crossed simplicial groups.

\section{Homology AND COHOMOLOGY OF $\Delta G$-MODULeS}

6.1. The notions of homology and cohomology of a group can be generalized to any small category. To define them we can use a standard resolution which is 
constructed below. It is well known that for a given group there may be a better resolution (example: cyclic and quaternion groups admit periodic resolutions). This is also the case for some categories (example $\Delta$, see below). More interesting for us is the case of $\Delta G$. Taking into account the fact that $\Delta G$ comes from a double category we show that there is a special biresolution which permits us to compute the homology and the cohomology via spectral sequences. This biresolution combines a resolution of $\Delta$ and the standard resolutions of the groups $G_{n}$. Another type of biresolution and a generalization of periodic cyclic homology to $H G_{*}$-theories can be found in [G].

6.2. In this section $k$ is a fixed commutative ring with 1 , referred to as the ground ring. If $X$ is a set, then $k[X]$ denotes the free $k$-module with basis $X$. We first recall the definition and properties of homology and cohomology of $\mathscr{C}$-modules, where $\mathscr{C}$ is a small category.

Let Mod be the category of $k$-modules. A $\mathscr{C}$-module is a functor from $\mathscr{C}$ to Mod. Let $N$ be a $\mathscr{C}$-module and $M$ be a $\mathscr{C}^{\text {op }}$-module. The tensor product $N \otimes_{\mathscr{C}} M$ is a $k$-module defined by

$$
N \otimes_{\mathscr{C}} M=\bigoplus_{C \in \mathrm{Ob} \mathscr{C}} N(C) \otimes_{k} M(C) / \sim,
$$

where the equivalence relation $\sim$ is generated by: for every morphism $f: C \rightarrow$ $D$ in $\mathscr{C}$, every $x \in N(C)$ and every $y \in M(D)$ we have $x \otimes f^{*}(y) \sim f_{*}(x) \otimes y$.

The category of $\mathscr{E}$-modules is abelian and has enough projectives and injectives. As the functor $N \otimes_{\mathscr{C}}$ - is right exact it admits derived functors denoted $\operatorname{Tor}_{n}^{\mathscr{C}}(N,-)$ such that $\operatorname{Tor}_{0}^{\mathscr{C}}(N, M)=N \otimes_{\mathscr{C}} M$.

Similarly, if $P$ is another $\mathscr{C}$-module, then $\operatorname{Hom}_{\mathscr{E}}(N, P)$ is a $k$-module and the derived functors of $\operatorname{Hom}_{\mathscr{C}}(N,-)$ are denoted $\operatorname{Ext}_{\mathscr{E}}^{n}(N,-)$.

6.3. The standard resolution. Let $k$ be the trivial $\mathscr{C}$-module; that is, every object has image $k$ and every morphism has image $i d_{k}$. We construct a free resolution of $k$ by $\mathscr{C}$-modules

$$
0 \leftarrow k \leftarrow L_{0} \leftarrow L_{1} \leftarrow L_{2} \leftarrow \cdots
$$

as follows. First we construct a simplicial $\mathscr{C}$-set $\left\{(\text { MOR } \mathscr{C})_{n}\right\}, L_{n}$ will be the free $k$-module on the set $\left.\{\operatorname{MOR} \mathscr{C})_{n}\right\}$ and $d=\sum(-1)^{i} d_{i}$ the boundary map.

Let MOR $\mathscr{C}$ be the category whose objects are the morphisms $f: A \rightarrow C$ of $\mathscr{C}$. A morphism in MOR $\mathscr{C}$ from $f$ to $g: B \rightarrow C$ (same $C$ as in $f$ ) is a morphism $h: A \rightarrow B$ in $\mathscr{C}$ such that $g \cdot h=f$. This category has a nerve which is the simplicial set $\left\{(\operatorname{MOR} \mathscr{C})_{n}\right\}$. In fact $\left\{(\operatorname{MOR} \mathscr{C})_{n}\right\}$ is a $\mathscr{C}$-set:

$$
C \rightarrow\left(A_{0} \rightarrow A_{1} \rightarrow \cdots \rightarrow A_{n} \rightarrow C\right)
$$

and it is easy to verify that $\left\{(\operatorname{MOR} \mathscr{C})_{n}\right\}$ is a simplicial $\mathscr{C}$-set.

Lemma 6.4. The complex $L_{*}$, where $L_{n}=k\left[(\operatorname{MOR} \mathscr{C})_{n}\right]$ and the boundary is $d=\sum(-1)^{i} d_{i}$, is a projective resolution of $\mathscr{C}$-modules of the trivial $\mathscr{C}$-module $k$. 
Proof. Let $s:(\operatorname{MOR} \mathscr{C})_{n} \rightarrow(\operatorname{MOR} \mathscr{C})_{n+1}$ be the map given by

$$
s\left(A_{0} \rightarrow A_{1} \rightarrow \cdots A_{n} \rightarrow C\right)=\left(A_{0} \rightarrow A_{1} \rightarrow \cdots \rightarrow A_{n} \rightarrow C \rightarrow C\right),
$$

where the last arrow is the identity of $C$. It induces a map of $k$-modules on $L_{n}$ that we multiply by $(-1)^{n+1}$ to get $s^{\prime}: L_{n} \rightarrow L_{n+1}$. It is immediate to verify that $d s^{\prime}+s^{\prime} d=i d$, which proves that $L_{*}$ is an acyclic complex. Hence the groups $\operatorname{Tor}_{n}^{\mathscr{E}}(k, M)$ are the homology groups of the complex of $k$-modules

$$
L_{0} \otimes_{\mathscr{C}} M \leftarrow L_{1} \otimes_{\mathscr{C}} M \leftarrow L_{2} \otimes_{\mathscr{C}} M \leftarrow \cdots .
$$

6.5. Example 1. $\mathscr{C}=G$. If $\mathscr{C}$ is the category with only one object and with morphisms the elements of a group $G$, a $G$-module in the above sense is a left $k[G]$-module and a $G^{\mathbf{o p}}$-module is a right $k[G]$-module. Therefore $\operatorname{Tor}_{n}^{G}(k, M)$ is the homology of the discrete group $G$ with coefficients in $M$, usually denoted by $H_{n}(G, M)$. The standard bar resolution described above is canonically isomorphic to the standard resolution described in [ML] via $\left(g_{0}, \ldots, g_{n}\right) \rightarrow$ $\left(g_{n} \ldots g_{0}, g_{n} \ldots g_{1}, \ldots, g_{n}\right)$.

6.6. Example 2. $\mathscr{C}=\Delta$. In this case there is a better resolution than the standard one. Put $\left(C^{m}\right)_{n}=$ free $k$-module on the set $\operatorname{Hom}_{\Delta}([m],[n])$. It is immediate that $C^{m}$ is a $\Delta$-module and that $C^{*}$ is a simplicial $\Delta$-module which gives rise to a resolution of the trivial $\Delta$-module $k$. Let $M$ be a simplicial module (i.e. a $\Delta^{\mathbf{o p}}$-module). The map $f \otimes x \rightarrow f^{*}(x)$ is an isomorphism $C^{m} \otimes_{\Delta} M \rightarrow M_{m}$ and therefore the homology of the classical complex

$$
M_{0} \leftarrow M_{1} \leftarrow M_{2} \leftarrow \cdots,
$$

where the boundary is $\sum(-1)^{i} d_{i}$, is exactly $\operatorname{Tor}_{n}^{\Delta}(k, M)$.

6.7. Example 3. $\mathscr{C}=\Delta G$. As $\Delta G$ is the category associated to the double category $\Delta G$ it is natural to look for a biresolution of the trivial $\Delta G$-module $k$. We could construct a standard biresolution associated to $\Delta \mathbf{G}$. However, we can take advantage of the existence of a simple resolution for $\Delta$. For $G_{n}$ we take the homogeneous bar resolution (see Example 1).

We first construct a bisimplicial $\Delta G$-set $\left\{\underline{T}^{p, q}\right\}$. Let $\underline{T}^{p, q}: \Delta G \rightarrow$ Sets be the functor which associates to $[n]$ the set $G_{q}^{(p+1)} \times \operatorname{Hom}_{\Delta \mathrm{G}}([q],[n])$. An element of this set will be denoted $\left(g_{0}, g_{1}, \ldots, g_{p}, \alpha\right)$. Define the bisimplical structure as follows:

$$
\begin{aligned}
& d_{i}^{h} \text { means omit } g_{i} . \\
& s_{i}^{h} \text { means repeat } g_{i} .
\end{aligned}
$$

$d_{i}^{v}, s_{i}^{v}$ means apply faces or degeneracies to each component.

Next define an action of $G_{q}$ on $k\left[\underline{T}^{p, q}\right]$ as follows:

$$
g\left(g_{0}, \ldots, g_{p}, \alpha\right)=\operatorname{sign}(g)\left(g g_{0}, \ldots, g g_{p}, \alpha \cdot g\right)
$$


where $\operatorname{sign}(g)$ denotes the sign of the permutation given by the action of $g$ on $[q]$. Define $\underline{C}^{p, q}$ to be the quotient of $k\left[\underline{T}^{p, q}\right]$ under this action. Now $C^{p, q}$ is no longer bisimplicial but only a bi-(chain complex). The reason is the following formula relating the group action to the vertical faces:

$$
(-1)^{i} d_{i}^{v}\left(g\left(g_{0}, \ldots, g_{p}, \alpha\right)\right)=(-1)^{g^{-1} i} d_{i}(g) d_{g^{-1} i}^{v}\left(g_{0}, \ldots, g_{p}, \alpha\right)
$$

which follows from the commutative diagram:

$$
\begin{array}{cc}
{[q-1] \stackrel{\delta_{g-1_{i}}}{\longrightarrow}[q]} \\
\downarrow d_{i}(g) & \downarrow g \\
{[q-1] \stackrel{\delta_{i}}{\longrightarrow}[q]}
\end{array}
$$

$\underline{C}^{p, q}$ is in fact a biresolution of the trivial $\Delta G$-module $k$. To see this note that the map which sends $\left(g_{0}, \ldots, g_{p}, \alpha\right)$ to $\left(g_{0}, \ldots, g_{p}, g, \alpha\right)$, where $\alpha=\phi \cdot g$ is the unique factorization of $\alpha$ with $\phi \in \Delta$ and $g \in G_{q}$, gives rise to a horizontal homotopy which proves acyclicity for $p \geq 0$.

Hence we have proved the following

Proposition 6.8. The bicomplex $C^{* *}$ described above is a biresolution of the trivial $\Delta G$-module $k$.

Notation. For the small category $\Delta G$ and the $\Delta G^{\text {op }}$-module $M$ we write $H G_{n}(M)$ in place of $\operatorname{Tor}_{n}^{\Delta G}(k, M)$ and $H G^{n}(M)$ in place of $\operatorname{Ext}_{\Delta G}^{n}\left(k, M^{*}\right)$. This agrees with the notation $H C_{n}$ used for cyclic homology and the notations $H D_{n}$ and $H Q_{n}$ for dihedral and quaternionic homologies. With this notation symmetric homology is denoted $H S_{n}(M)$. We also denote by $\bar{M}_{q}$ the $G_{q}$-module $M_{q}$ with the action of $G_{q}$ twisted by sign .

From a bicomplex whose homology gives $H G_{n}(M)$ one can deduce two spectral sequences. One of them reads as follows.

Theorem 6.9. Let $M$ be a $\Delta G^{\mathrm{op}}$-module. There is a first quadrant spectral sequence

$$
E_{p q}^{1}=H_{p}\left(G_{q}, \bar{M}_{q}\right) \Rightarrow H G_{p+q}(M)
$$

whose differential $d^{1}$ is induced by the simplicial boundary $d=\sum(-1)^{i} d_{i}$.

Proof. Consider the biresolution $C^{* *}$ of $k$ given in 6.8. It gives rise to a bicomplex which computes $H G_{n}(M)$. Horizontally the homology is the homology of the discrete group $G_{q}$ with coefficients in the $G_{q}$-module $\bar{M}_{q}$. This is the $E_{p q}^{1}$ term. The differential $d^{1}$ is induced by the vertical differential which is precisely $d$.

Corollary 6.10. If all the groups $G_{n}$ are finite and if $k$ contains $\mathbf{Q}$, the group $H G_{n}(M)$ is isomorphic to the homology group of the complex of coinvariants $\left(\left(\bar{M}_{n}\right)_{G_{n}}, d\right)$.

Proof. In the spectral sequence $E_{p q}^{1}=0$ for $p \neq 0$ and $E_{0 n}^{1}=\left(M_{n}\right)_{G_{n}}$. 
6.11. (Co)-homology with coefficients in a free module. Let $\mathscr{C}$ be a small category and $X$ a functor from $\mathscr{C}^{\text {op }}$ to Sets. The homotopy colimit of $X$ is a space denoted hocolim $X$ whose definition is recalled below. If we compose $X$ with the functor which assigns to a set the free $k$-module on this set we get a $\mathscr{C}^{\text {op }}$-module $k[X]$.

Theorem 6.12. For any functor $X: \mathscr{C}^{\mathrm{op}} \rightarrow$ Sets there are canonical isomorphisms

$$
\operatorname{Tor}_{n}^{\mathscr{E}}(k, k[X])=H_{n}(\text { hocolim } X, k)
$$

and

$$
\operatorname{Ext}_{\mathscr{E}}^{n}\left(k,(k[X])^{*}\right)=H^{n}(\text { hocolim } X, k) .
$$

Proof. To compute the homology of hocolim $X$ with coefficients in $k$ one can form a simplicial module out of the nerve of $\mathscr{C}_{X}$ by taking the free module over the $n$-simplices in each dimension. The complex of modules deduced from this simplicial module has $H_{n}($ hocolim $X, k)$ for homology groups.

On the other hand if we choose the standard resolution described in [B-K] to compute $\operatorname{Tor}_{n}^{\mathscr{C}}(k, k[x])$ we find exactly the same complex as above. This ends the proof of the theorem.

Corollary 6.13. Let $X_{*}$ be a $G_{*}$-set and $k[X]$ its associated $\Delta G^{0 \mathbf{p}}$-module. Then $H G_{*}(k[X])=H_{*}\left(E\left|G_{*}\right| \times_{\mid G_{*}}\left|X_{*}\right|\right)$ and the (second) spectral sequence associated to the bicomplex 6.7 is the spectral sequence of the fibration

$$
\left|G_{*}\right| \rightarrow\left|X_{*}\right| \rightarrow E\left|G_{*}\right| \times_{\left|G_{*}\right|}\left|X_{*}\right| \text {. }
$$

Proof. This is an immediate application of 5.12 and 6.12 .

6.14. Examples of $\Delta G$-modules. Let $A$ be an associative $k$-algebra (not necessarily commutative) and let $A^{\otimes n}$ be the $n$-fold tensor product of $A$ over $k$. There is a canonical structure of $\Delta^{\mathrm{op}}$-module on the $A^{\otimes n+1}$,s given by $[n] \rightarrow A^{\otimes n+1}$,

$$
\begin{aligned}
d_{i}\left(a_{0}, \ldots, a_{n}\right) & =\left(a_{0}, \ldots, a_{i} a_{i+1}, \ldots, a_{n}\right) \text { for } 0 \leq i \leq n-1, \\
d_{n}\left(a_{0}, \ldots, a_{n}\right) & =\left(a_{n} a_{0}, \ldots, a_{n-1}\right), \\
s_{i}\left(a_{0}, \ldots, a_{n}\right) & =\left(a_{0}, \ldots, a_{i}, 1, a_{i+1}, \ldots, a_{n}\right) \text { for } 0 \leq i \leq n .
\end{aligned}
$$

The generator $t_{n}$ of $\mathbf{Z} / n+1$ acts on $A^{\otimes n+1}$ by

$$
t_{n}\left(a_{0}, \ldots, a_{n}\right)=\left(a_{n}, a_{0}, \ldots, a_{n-1}\right) \text {. }
$$

These actions give rise to a $\Delta G^{\mathrm{op}}$-module as shown in [C]. The associated homology is called cyclic homology and is denoted $H C_{*}(A)$ (cf. [L-Q]).

If the algebra $A$ is endowed with an involution $a \mapsto \bar{a}$ such that $\overline{a b}=\bar{b} \bar{a}$, then $[n] \mapsto A^{\otimes n+1}$ is in fact a $\Delta D^{\mathbf{o p}}$-module (and hence a $\Delta Q^{\mathbf{o p}}$-module). The action of the generator $x$ of $D_{n+1}$ is as $t_{n+1}$ above and the action of the generator $y$ is given by

$$
y\left(a_{0}, \ldots, a_{n}\right)=(-1)^{n(n-1) / 2}\left(\bar{a}_{0}, \bar{a}_{n}, \ldots, \bar{a}_{1}\right) .
$$


This example is studied in detail in [L], where the associated homology $H D_{*}(A)$ (resp. $\left.H Q_{*}(A)\right)$ is called dihedral homology (resp. quaternionic homology).

Note that $[n] \mapsto A^{\otimes n+1}$ is not a $\Delta S^{\text {op }}$-module; however $[n] \mapsto k\left[S_{n+1}\right] \otimes A^{\otimes n+1}$ can be endowed with a structure of $\Delta S^{\mathrm{op}}$-module.

6.15. Symmetric homology. The comparison between cyclic homology and Hochschild homology gives rise to the "periodicity exact sequence," which is due to the fact that $\left|C_{*}\right|=S^{1}$. In the symmetric, hyperoctahedral and braid cases we take advantage of the fact that there exists a null homotopy (cf. 1.5, Example 6) or equivalently that $\left|S_{*}\right|,\left|H_{*}\right|$ and $\left|B_{*}\right|$ are contractible.

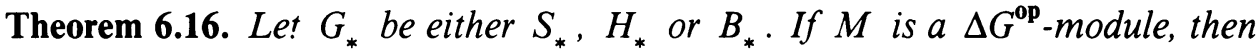
the canonical map $H_{n}(M) \rightarrow H G_{n}(M)$ is an isomorphism.

Proof. Consider the resolution constructed in 6.7. The homology $H G_{*}(M)$ is the homology of the double complex $\mathscr{C}^{p, q} \otimes_{\Delta G} M=k\left[\left(G_{q}\right)^{p}\right] \otimes_{k} M_{q}$.

Let $p$ be fixed for a moment and put $X_{q}=k\left[\left(G_{q}\right)^{p}\right]$. The map $X_{q} \rightarrow X_{q+1}$ defined by $\left(g_{1}, \ldots, g_{p}\right) \mapsto\left(h\left(g_{1}\right), \ldots, h\left(g_{p}\right)\right)$, where $h: G_{q} \mapsto G_{q+1}$ is the standard inclusion, is a homotopy; that is $d_{i} h=h d_{i}$ for $0 \leq i \leq q$ and $d_{q+1}=\mathrm{id}$.

In order to compute the homology of the complex $\left(X_{q} \otimes M_{q}\right)_{q \geq 0}$ we consider the simplicial modules $X_{*}, M_{*}$ and the resulting bicomplex $\left(X_{r} \otimes M_{s}\right)_{r, s>0}$. By the Eilenberg-Zilber-Cartier theorem the homology of the diagonal complex (which is the one we want to compute) is the abutment of a spectral sequence. Because of the acyclicity of $X_{*}$ only $E_{0 s}^{2}$ is nonzero and it is in fact $H_{s}\left(M_{*}\right)$. Therefore $H_{s}\left(M_{*}\right) \rightarrow H_{s}\left(\left(X_{q} \otimes M_{q}\right)_{q \geq 0}\right)$ is an isomorphism.

This proves that the map of bicomplexes $\mathscr{C}^{p, q} \otimes_{\Delta} M \rightarrow \mathscr{C}^{p, q} \otimes_{\Delta G} M$ is a quasi-isomorphism when restricted to columns. Therefore $H_{*}(M) \rightarrow H G_{*}(M)$ is an isomorphism.

Corollary 6.17. Let $k$ be a characteristic zero field and let $M$ be a $\Delta S^{\text {op }}$. module. The surjection of complexes $\left(M_{n}, d\right)_{n \geq 0} \rightarrow\left(\left(\bar{M}_{n}\right)_{S_{n}}, d\right)_{n \geq 0}$ is a quasiisomorphism (also true for $H_{*}$ in place of $S_{*}$ ).

Proof. This is an immediate consequence of 6.16 and 6.10 .

\section{REFERENCES}

[A] R. Aboughazi, Groupes simpliciaux croisés symétriques et hyperoctahédral, Preprint, IRMA, Strasbourg, 1986.

[B-K] A. K. Bousfield and D. M. Kan, Homotopy limits, completions and localizations, Lecture Notes in Math., vol. 304, Springer-Verlag, 1972.

[B-F] D. Burghelea and Z. Fiedorowicz, Cyclic homology and algebraic $K$-theory of spaces. II, Topology 25 (1986), 303-317.

[C] A. Connes, Cohomologie cyclique et foncteurs $\mathrm{Ext}^{n}$, C. R. Acad. Sci. Paris 296 (1983), 953958. 
[D-K] W. G. Dwyer and D. M. Kan Normalizing the cyclic modules of Connes, Comment. Math. Helv. 60 (1985), 582-600.

[G] F. Goichot, Homologie cyclique: produits, généralisations, Preprint, IRMA, Strasbourg, 1986.

[L] J-L. Loday, Homologies diédrale et quaternionique, Adv. in Math. 66 (1987), 119-148.

[L-Q] J-L. Loday and D. Quillen, Cyclic homology and the Lie algebra homology of matrices, Comment. Math. Helv. 59 (1984), 565-591.

[ML] S. Mac Lane, Categories for the working mathematician, Graduate Texts in Math., SpringerVerlag, 1971.

[Q] D. Quillen, Higher algebraic $K$-theory, Algebraic $K$-theory. I, Lecture Notes in Math., vol. 341, Springer-Verlag, 1972, pp. 85-147.

[R] M. S. Ragunathan, Discrete subgroups of Lie groups, Ergeb. der Math. Grenzgeb., SpringerVerlag, 1972.

Department of Mathematics, Ohio State University, Columbus, Ohio 43210

Institut de Recherche Mathématique Avancée, CNRS, 67084 Strasbourg, France 